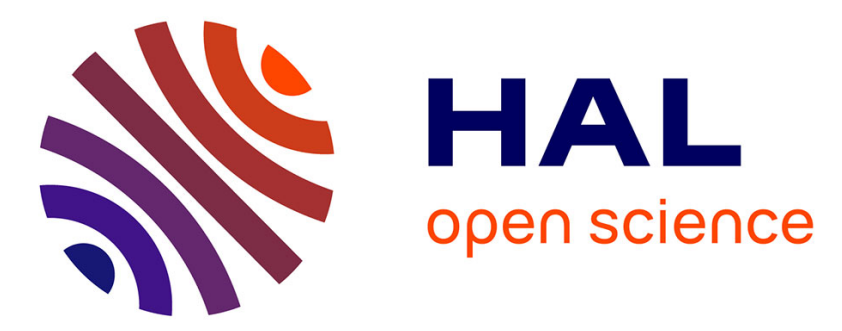

\title{
Micro/macro viability analysis of individual-based models: Investigation into the viability of a stylized agricultural cooperative
}

Sophie Martin, Isabelle Alvarez, Jean-Daniel Kant

\section{- To cite this version:}

Sophie Martin, Isabelle Alvarez, Jean-Daniel Kant. Micro/macro viability analysis of individual-based models: Investigation into the viability of a stylized agricultural cooperative. Complexity, 2015, 21 (2), pp.276-296. 10.1002/cplx.21604 . hal-01340599

\author{
HAL Id: hal-01340599 \\ https://hal.science/hal-01340599
}

Submitted on 5 Apr 2017

HAL is a multi-disciplinary open access archive for the deposit and dissemination of scientific research documents, whether they are published or not. The documents may come from teaching and research institutions in France or abroad, or from public or private research centers.
L'archive ouverte pluridisciplinaire HAL, est destinée au dépôt et à la diffusion de documents scientifiques de niveau recherche, publiés ou non, émanant des établissements d'enseignement et de recherche français ou étrangers, des laboratoires publics ou privés. 


\title{
Micro/Macro Viability Analysis of Individual-based Models: Investigation into the Viability of a Stylized Agricultural Cooperative
}

\author{
S. Martin ${ }^{\mathrm{a}}$, I. Alvarez ${ }^{\mathrm{a}, \mathrm{b}}$, J.-D. Kant ${ }^{\mathrm{b}}$ \\ ${ }^{a}$ LISC, Irstea, 24 avenue des Landais \\ BP 50085, 63172 Aubière, France \\ ${ }^{b}$ LIP6, Université Pierre et Marie Curie, 4 place Jussieu \\ 75252 Paris cedex 05, France
}

\begin{abstract}
The mathematical viability theory proposes methods and tools to study at a global level how controlled dynamical systems can be confined in a desirable subset of the state space. Multi-level viability problems are rarely studied since they induce combinatorial explosion (the set of $N$ agents each evolving in a $p$-dimensional state space, can evolve in a $N p$ dimensional state space). In this paper, we propose an original approach which consists in solving first local viability problems and then studying the real viability of the combination of the local strategies, by simulation where necessary. In this article we consider as multi-level viability problem a stylized agricultural cooperative which has to keep a minimum of members. Members have an economical constraint and some members have a simple model of the functioning of the cooperative and make assumptions on other members' behavior, especially pro-viable agents which are concerned about their own viability. In this framework, the model assumptions allow us to solve the local viability problem at the agent level. At the cooperative level, considering mixture of agents, simulation results indicate if and when including pro-viable agents increases the viability of the whole cooperative.
\end{abstract}

Keywords: Viability theory, individual-based models, local/global viability problems 2000 MSC: 37N35, 93B03, 93B52, 37M05 


\section{Introduction}

For Hardin [1], common goods are doomed to be overexploited and devastated. Noting that there exists thousands of practical examples preventing the "tragedy of the commons" in reality, Ostrom [2] prefers to address the "issue of how to improve the skills of participants to change the rules of the game coercive to achieve results different from the relentless tragedies". Schuster [3] considers that no matter how nice the successful examples of selfgovernance are we should also not forget the large numbers of cases where self-organization failed terribly and his conclusion is that the evolution of interactions between governmental interference and self-governance is still not sufficiently well understood yet.

Mathematical methods and models can help to understand the general processes of interactions and regulations happening in groups made of individuals. The complex system is viewed as a network of interacting discrete entities (individuals or objects) and individual-based model group includes models ranging from cellular automata to very detailed agent-based models on various social network graphs. These individual-based models are becoming the tools of choice for investigating the behavior of groups of individuals in many fields $[4,5]$ : Scheffran and Hannon [6] introduce a general framework for modeling the interactions between agents in which agents can adjust their actions and resources to those of other agents and study by simulations the transition from conflict to cooperation in several cases; considering a model of groundwater use, López et al. [7] show that the success of the optimal management program depends heavily on the information that the users have about the resource.

The problem addressed in this paper is the investigation of the links between microscopic/individual viability and macroscopic/global viability in individual-based mathematical models. The issue is the persistence of a group and the entities that make it up over time.

The mathematical viability theory has been developing for thirty years methods and tools to study the compatibility between dynamics and constraints $[8,9]$. This framework has been applied to renewable resources management and especially to the regulation of fisheries [10,11, 12, 13], forest preservation $[14,15]$ or lake eutrophication management [16] as well as to broader(eco)-system dynamics $[17,18,19]$ or to pure economic or social ones $[20,21]$.

In all these works, systems are described by global variables and the con- 
trol regulation also operates at the global scale, for example the total amount of harvest or the total amount of phosphorus inputs in the lake. However, the total amount of harvest is the sum of harvest of individual fisheries, as the total amount of phosphorus inputs is the sum of phosphorus inputs of individual farmers. And these entities may have their own dynamics and constraints at a local scale. Viability studies including macro and micro scales are rare. Studying quotas in marine fisheries, Péreau et al. [22] recently determined viable global quotas strategies integrating a constraint at the global level which takes into account the social and economic constraints at the level of individual fisheries (considering the less efficient). Considering agents harvesting a renewable resource, Doyen and Péreau [23] considered local controls (the effort of each agent $i$ ) but the viability problem they solve only concerns the global level.

As far as individual-based models are concerned, viability constraints can both rely on local and global scales. Once agent behaviors and interaction rules are defined, the evolution of the system can be analysed by statistical mechanics techniques (master equation in the mean-field limit in [24] for instance) or shown by extensive computer simulations as in [25].

The inverse problem of the individual strategy design is addressed by differential game theory: Every agent considered as a player chooses a control to maximize independently from the others his own performance objective. In the zero-sum differential games framework with two players, Bettiol et al. [26] have included state constraints; nonzero-sum differential games with a very large number of players have been investigated in the terminology of mean-field games [27, 28]. But the advances on nonzero-sum differential games with $N$ players have been scarcer, and mainly restricted to linear quadratic games for the main reason that there was very little understanding of the system of Hamilton-Jacobi equations naturally attached to these games [29].

In this article, we then propose to combine viability theory and simulations to study viability properties of an individual based model ( $N$ players in game theory terminology) facing both local and global constraints: We use viability theory tools to derive viable local individual strategies and simulations to study the impact of these strategies on collective viability.

Agricultural cooperatives are world-wide well-known farmers organizations (see for instance [30] for German cooperatives). An agricultural cooperative and its members may constitute a relatively simple example of two 
scales systems: They are characterized by open membership societies, collective ownership, equal voting power, principles of equality and solidarity. This cooperative model is constructed for making cooperative business efficient under specific economic conditions especially when the average cost curve is continually declining with size [31]. Cooperative members, however, are rather heterogeneous. In general, members can be differentiated according to geographic dispersion, variances in age and education, farm size and type, as well as business objectives and strategies. Furthermore, when members do not see a strong connection between the success of the cooperative and their own business, Fulton and Giannakas [32] showed that member heterogeneity concerning the trust and loyalty toward the cooperative firm may lead to substantial financial pressures for the cooperatives. For example in wine cooperatives, usually the same price is paid for the same grape. Therefore, each viticulturist that belongs to a wine cooperative can produce the grapes for every wine that he wishes, regardless whether the grapes match the consumer quality criteria in taste. As a result, members select their grapes adversely so that they sell the better-quality grapes to other mostly private owned enterprises [33].

Several works apply economic theory to agricultural cooperative, they develop models using neoclassical theory solving members' utility maximization problem to find optimal pricing rules or game theory for instance to examine the issue of member commitment in the context of a mixed oligopoly where cooperatives and investor-owned firms compete with each other in supplying a consumer good [34]. In this article we build a model that includes the evolution with time of the cooperative and its members to be able to study the viability of this two scales system that is its persistence over time.

In the stylized cooperative we propose to study, we consider that members heterogeneous behavior is described by their loyalty toward the cooperative: The $N$ members of the cooperative earn an amount proportional to the provided quantity. They have the possibility to fraud by increasing artificially the provided amount without being discovered until a given threshold. However, the proportionality coefficient used to evaluate their earnings depends on the global quality of the cooperative production which is altered by fraud.

From a viability viewpoint, if the cooperative members get no information and are not subjected to any pressure from the aggregated level, it is then only a juxtaposition of individual independent local viability problems (as it is rather the case in consumer's buying groups). When the global level makes the local controls compulsory, then it is a classical global viability problem. 
In this paper we consider a partial influence of the global level on the local level: The payment of each member depends on the average level of fraud.

In this framework we study the impact of the individual local strategies on the viability at the global level. A naive approach consists in considering the viability problem of the global system of $N$ agents. In general it is impossible to solve: It deals with a dynamical system of at least $N$ state variables. Moreover, it is very often unrealistic to assume that the global level can enforce the behavior of each agent. In order to make the problem tractable, we propose to provide each agent with a model of the impact of the other members' behavior on its income. Making assumptions about the other members' behavior, the agent is able to solve its own local viability problem. Here we consider simple models and behaviors, and very elementary hypothesis about the possible behaviors. The objective of the paper is to show that even in this stylized framework, individual viability concerns can generate collective viability.

The paper is organized as follows: In the next section we present the particular viability problem we propose to address. In section three we describe the illustrative cooperative model from which we derive local viability problems depending on three agent types. We present the theoretical results concerning the viability of the strategies followed by the three types of agents in section four. The results concerning the global viability of the whole cooperative are described in section five. Finally, the main conclusions of this work are summarized in section six.

\section{Problem statement: Interlinked local viability problems}

We consider $N$ agents, members of the same global entity.

Each agent $i$ is characterized by its own individual state variables, $x_{i} \in X_{i} \subset$ $R^{n_{i}}$, and control variables, $u_{i} \in R^{p_{i}}$. Since all agents are members of the same global entity, each of the individual dynamics depends on the individual state and control variables but it is also influenced by the state variables of the global entity $x \in X \subset R^{n}$ :

$$
\begin{aligned}
& x_{i}^{\prime}(t)=f_{i}\left(x_{i}(t), u_{i}(t), x(t)\right) \\
& u_{i}(t) \in U_{i}\left(x_{i}(t), x(t)\right)
\end{aligned}
$$

where $U_{i}\left(x_{i}, x\right) \subset R^{p_{i}}$ is the set of admissible controls for agent $i$ when its state is $x_{i}$ and the global entity state is $x$. Local agents face local viability 
constraints described by $L_{i} \subset X_{i}$ :

$$
\forall t, \quad x_{i}(t) \in L_{i} .
$$

Moreover, the variations of the macroscopic variables $x \in X \subset R^{n}$ describing the global entity depend on their own values but also on local variables and controls :

$$
x^{\prime}(t)=f\left(x(t),\left\{x_{i}(t)\right\}_{1, \ldots, N},\left\{u_{i}(t)\right\}_{1, \ldots, N}\right) .
$$

To solve a viability problem is to find control strategies such that all the constraints are satisfied over time. Here the dimension of the state space equals $\sum_{1}^{N} n_{i}+n$, so the classical approach of [8] is intractable. We then propose to study this problem in two steps. First we solve the viability problems at the local level, allowing each agent to make assumptions on the evolution of the other members' behavior. With these hypotheses, it is possible to compute for each agent viable strategies for its own local viability problem. Second, we propose to study by simulation the real viability of the combination of these local strategies when they are implemented by the agents.

\section{The case study: The cooperative and the micro/macro viability problem}

\subsection{The model of the cooperative}

In our stylized cooperative, the cooperative is described by a single global variable which is the price at which the production is sold; each member has one state variable, its capital, and one control variable, its fraud level. However, the evolution with time of all these variables are correlated that makes the local and global viability issues intertwined.

\subsubsection{The agent model}

Each agent $i$ has only one state variable: Its capital $K_{i}$, which evolves with the simple following dynamics:

(i) Each agent $i$ produces a quantity $w_{i}$.

(ii) Each agent $i$ can control partially the quality of its production. Here we consider that agents can alter the quality by fraud, which means 
artificially increasing the production quantity brought to the cooperative. Actually, each member brings a quantity $\alpha_{i} w_{i}$ to the cooperative, $\alpha_{i}=1$ if he does not fraud and $\left.\left.\alpha_{i} \in\right] 1 ; \bar{\alpha}\right]$ otherwise. The value $\bar{\alpha}$ corresponds to the maximal fraud level which avoids detection.

(iii) Each member $i$ pays fix charges with amount $C_{i}$ associated with its production activity including the minimal salary for its subsistence; Each member benefit equals $p \alpha_{i} w_{i}-C_{i}$ where $p$ is the price per quantity unit paid by the cooperative to all members.

The capital, $K_{i}(t)$, of each member $i$ evolves with time according to its benefits:

$$
K_{i}(t+1)=K_{i}(t)+p(t+1) \alpha_{i}(t+1) w_{i}(t+1)-C_{i}(t+1) .
$$

\subsubsection{The global price dynamics}

We consider a single global variable: The price per quantity unit $p(t)$. This price depends on:

(i) The cooperative production quality, $Q$, which depends at each time step on the global fraud amount that can be measured by comparing $\sum_{i} \alpha_{i} w_{i}$ and $\sum_{i} w_{i}$.

If no member frauds, $\sum_{i} \alpha_{i} w_{i}=\sum_{i} w_{i}$ and the global cooperative production quality is high equal to $Q_{0}$. However, when some members fraud, the quality is lower. If all members fraud with highest value $\bar{\alpha}$, $\sum_{i} \alpha_{i} w_{i}=\bar{\alpha} \sum_{i} w_{i}$ and the quality of the production of the cooperative is the worst quality the cooperative can produce, $Q$. We consider here that a highly degraded global quality results in a decrease of individual incomes. Indeed, if the price is proportional to the quality, the individual income is proportional to the product of the global quality by the individual production. This income equals $Q_{0} . w_{i}$ if no member frauds. When all members fraud with highest value $\bar{\alpha}$, the global production is $\bar{\alpha} \sum_{i} w_{i}$, but the quality is degraded in a higher proportion we set to $\underline{Q}=Q_{0} / \bar{\alpha}^{2}$. Hence the individual income is lower if all the members

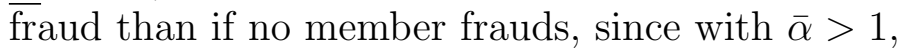

$$
\frac{Q_{0}}{\bar{\alpha}^{2}} \cdot \bar{\alpha} \cdot w_{i}=\frac{1}{\bar{\alpha}} Q_{0} \cdot w_{i}<Q_{0} \cdot w_{i} .
$$

Once set the extreme values, we assume that the quality, $Q$, varies linearly with the global fraud amount $\frac{\sum_{i} \alpha_{i} w_{i}}{\sum_{i} w_{i}}$. And consequently, 


$$
Q=-Q_{0} A \frac{\sum_{i} \alpha_{i} w_{i}}{\sum_{i} w_{i}}+Q_{0}(1+A)
$$

with

$$
A=\frac{\bar{\alpha}+1}{\bar{\alpha}^{2}} .
$$

Actually, $Q\left(\sum_{i} \alpha_{i} w_{i}=\bar{\alpha} \sum_{i} w_{i}\right)=\frac{Q_{0}}{\bar{\alpha}^{2}}$ (i.e. maximal fraud) and $Q\left(\sum_{i} \alpha_{i} w_{i}=\right.$ $\left.\sum_{i} w_{i}\right)=Q_{0}$ (no fraud).

(ii) The cooperative reputation: When the quality decreases, the effect is instantaneous, the price per quantity unit paid to each member corresponds to the global production quality of the cooperative; However, when the quality increases, the price does not increase as fast as it has decreased, it is a matter of reputation. The effect of a quality decrease is instantaneous, so we set that $\Delta p(t):=p(t+1)-p(t)$ is proportional to $\Delta Q(t):=Q(t+1)-Q(t)$. When the quality increases, that is $\Delta Q(t) \geq 0$, the price will increase less quickly since getting a good reputation takes time. This inertia is represented by the weight $\delta \in[0 ; 1[$ allocated to the previous price, $p(t)$, when evaluating $p(t+1)$ :

$$
\begin{array}{ll}
p(t+1)=p(t)+\frac{p_{0}}{Q_{0}} \Delta Q(t) & \text { if } \Delta Q(t) \leq 0 \\
p(t+1)=\delta p(t)+(1-\delta)\left(p(t)+\frac{p_{0}}{Q_{0}} \Delta Q(t)\right) & \text { if } \Delta Q(t) \geq 0
\end{array}
$$

with $\frac{p_{0}}{Q_{0}}$ the proportionality coefficient: $p_{0}$ is the price corresponding to the highest quality $Q_{0}$.

From (5),

$$
\Delta Q=-Q_{0} A \Delta\left(\frac{\sum_{i} \alpha_{i} w_{i}}{\sum_{i} w_{i}}\right) .
$$

Consequently, from (7),

$$
\begin{aligned}
& p(t+1)=p(t)-p_{0} A \Delta\left(\frac{\sum_{i} \alpha_{i}(t) w_{i}(t)}{\sum_{i} w_{i}(t)}\right), \text { if } \Delta\left(\frac{\sum_{i} \alpha_{i}(t) w_{i}(t)}{\sum_{i} w_{i}(t)}\right) \geq 0 \\
& p(t+1)=p(t)-(1-\delta) p_{0} A \Delta\left(\frac{\sum_{i} \alpha_{i}(t) w_{i}(t)}{\sum_{i} w_{i}(t)}\right), \text { if } \Delta\left(\frac{\sum_{i} \alpha_{i}(t) w_{i}(t)}{\sum_{i} w_{i}(t)}\right) \leq 0
\end{aligned}
$$

\subsection{The model of the other members' behavior}

We provide the agents with a simple assumption on the other members' future behavior based on the idea that other agents may probably do the same reasoning as they does and then choose the same fraud level and others 
will behave in average as in the previous period: Agent $i$ anticipates that if it chooses for its next fraud level the value $\alpha(t+1)$, the next average fraud level of the whole cooperative measured by $\frac{\sum_{i} \alpha_{i}(t+1) w_{i}(t+1)}{\sum_{i} w_{i}(t+1)}$ will be equal to

$$
\frac{1 / B_{i}\left(\sum_{j=1}^{N} w_{j}(t+1)\right) \alpha_{i}(t+1)+\left(1-1 / B_{i}\right)\left(\sum_{j=1}^{N} w_{j}(t+1)\right) \alpha_{m}(t)}{\sum_{j=1}^{N} w_{j}(t+1)}
$$

with $B_{i}>1$.

Member $i$ anticipates that in the mean, at time $t+1$, other members will behave as if its new fraud level, $\alpha_{i}(t+1)$, would apply to a proportion $1 / B_{i}$ of the global production $\left(\sum_{j=1}^{N} w_{j}(t+1)\right)$; Whereas the remaining proportion, $1-1 / B_{i}$, of the global production would experience a fraud level equal to the previous fraud average, $\alpha_{m}(t)$.

The agents estimate the previous fraud average, $\alpha_{m}(t)$, from the price, $p(t)$, they have sold their last production: if $p(t)=p_{0}$, then they estimate $\alpha_{m}(t)=1$, if $p(t)=p_{0} / \bar{\alpha}^{2}$, then they estimate $\alpha_{m}(t)=\bar{\alpha}$, and in general using a linear extrapolation:

$$
\alpha_{m}(t)=\frac{p_{0}(1+A)-p(t)}{p_{0} A} .
$$

Consequently, member $i$ anticipates that:

$$
\begin{aligned}
\Delta\left(\frac{\sum_{j=1}^{N} \alpha_{j}(t) w_{j}(t)}{\sum_{j=1}^{N} w_{j}(t)}\right) & =\frac{1 / B_{i} \sum_{j=1}^{N} w_{j}(t+1) \alpha_{i}(t+1)+\left(1-1 / B_{i}\right) \sum_{j=1}^{N} w_{j}(t+1) \alpha_{m}(t)}{\sum_{j=1}^{N} w_{j}(t+1)}-\alpha_{m}(t) \\
& =\frac{1}{B_{i}}\left(\alpha_{i}(t+1)-\alpha_{m}(t)\right) \\
& =\frac{1}{B_{i}}\left(\alpha_{i}(t+1)-\frac{p_{0}(1+A)-p(t)}{p_{0} A}\right) .
\end{aligned}
$$

\subsection{The local viability problems}

With these models of cooperative functioning and other members' behavior, $i$ local viability problems can be defined.

\subsubsection{The dynamics}

From (9) and (11), the global price value forecast by member $i$ equals: 


$$
\begin{aligned}
p(t+1)= & p(t)-\frac{p_{0} A}{B_{i}}\left(\alpha_{i}(t+1)-\frac{p_{0}(1+A)-p(t)}{p_{0} A}\right), \\
& \quad \text { if }\left(\alpha_{i}(t+1)-\frac{p_{0}(1+A)-p(t)}{p_{0} A}\right) \geq 0 \\
p(t+1)= & p(t)-(1-\delta) \frac{p_{0} A}{B_{i}}\left(\alpha_{i}(t+1)-\frac{p_{0}(1+A)-p(t)}{p_{0} A}\right), \\
& \quad \text { if }\left(\alpha_{i}(t+1)-\frac{p_{0}(1+A)-p(t)}{p_{0} A}\right) \leq 0 .
\end{aligned}
$$

Equations (4) and (12) give the values of the global price and of the capital of member $i$ at time $t+1$ as a function of their values at time $t$ and of member $i$ fraud value, $\alpha_{i}(t+1)$. Member $i$ chooses is next fraud level between 1 and $\bar{\alpha}$. Thus, let $u(t) \in[1 ; \bar{\alpha}]$,

$$
\alpha(t+1)=u(t) \in[1 ; \bar{\alpha}] .
$$

Variable $u$ is called control variable in the mathematical viability theory framework and the model of agent $i$, member of the cooperative, is a control dynamical system discrete with time.

The dynamics of each local viability problem is the following:

$$
\left\{\begin{array}{l}
K_{i}(t+1)=K_{i}(t)+p(t+1) u_{i}(t) w_{i}(t+1)-C_{i}(t+1) \\
p(t+1)=p(t)-\left(1-\delta * 1_{u_{i}(t)-\frac{\left(p_{0}(1+A)-p(t)\right)}{p_{0} A} \leq 0}\right) \frac{p_{0} A}{B_{i}}\left(u_{i}(t)-\frac{\left(p_{0}(1+A)-p(t)\right)}{p_{0} A}\right) \\
u_{i}(t)
\end{array}\right.
$$

with $1_{u_{i}(t)-\frac{\left(p_{0}(1+A)-p(t)\right)}{p_{0} A} \leq 0}=1$ when $u_{i}(t)-\frac{\left(p_{0}(1+A)-p(t)\right)}{p_{0} A} \leq 0$ and 0 otherwise.

For sake of simplicity, we consider in the following sections that $w_{i}(t)=$ $w_{i}$ and $C_{i}(t)=C_{i}$, to reduce the dimension of the dynamical system to 2 state variables $K_{i}$ and $p$. Otherwise it would be necessary to add the time dimension.

\subsubsection{The constraints}

The constraint on the global variable, $p(t) \in\left[\frac{p_{0}}{\bar{\alpha}^{2}} ; p_{0}\right]$ is necessarily satisfied for all $t \in \mathbb{N}$ according to global price evolution equation (14).

The simplest constraint is imposed on the capital of each individual member, $K_{i}$, which is to remain above a given threshold, $K_{i_{m i n}}$, with time, that is:

$$
\forall t \in \mathbb{N}, \quad K_{i}(t) \geq K_{i_{\min }} .
$$

This second constraint is not necessarily satisfied, it depends on the price the cooperative can sell its production which depends itself on the quality of the production and on the successive fraud amounts. 
The constraint set is then defined by:

$$
L_{i} \subset \mathbb{R}^{2}:=\left\{( K _ { i } , p ) \in \left[K_{i_{m i n}} ;+\infty\left[\times\left[\frac{p_{0}}{\bar{\alpha}^{2}} ; p_{0}\right]\right\} .\right.\right.
$$

\subsection{Different individual strategies}

In this paper, we aim at showing that individual viability concerns can generate collective viability. We will then consider three agent types:

1. Myopic agent replicates the previous average fraud level;

2. Maximizing agent maximizes its income;

3. Pro-viable agent maximizes its income among the viable fraud levels.

It is worth noticing that the first type of agent does not need any assumption on the other members' behavior, whereas the second and third ones do. Obviously many other types of agents and strategies could be studied.

\section{Theoretical results}

The myopic agents situation is easy to describe: The fraud level remains constant. Theoretical results concerns the maximal and the maximal viable individual strategies. In the following, we omit index $i$ in $K_{i}(t), u_{i}(t), B_{i}$, $w_{i}, C_{i}, K_{i_{\min }}$ and $L_{i}$.

\subsection{The strategy of maximizing agents does not always correspond to maxi-} mal fraud

From (14), $K(t+1)-K(t)$ depends on $p(t)$ and $u(t)$ but not on $K(t)$, so the strategy of the maximizing agent is described by the function, $u_{M}$, which only depends on $p$ :

$$
\begin{aligned}
u_{M}:\left[\frac{p_{0}}{\bar{\alpha}^{2}} ; p_{0}\right] & \rightarrow[1, \bar{\alpha}] \\
p & \rightarrow \operatorname{argmax}_{u \in[1 ; \bar{\alpha}]} K(t+1)-K(t)
\end{aligned}
$$

We also define the function, $\Delta p_{M}:\left[p_{0} / \bar{\alpha}^{2} ; p_{0}\right] \rightarrow \mathbb{R}$, which is the variation of $p$ when $u=u_{M}(p)$, which also only depends on $p$.

The following propositions specify the link between $u_{M}(p)$ and $p$ and assess sufficient conditions for the maximal strategy not to be viable.

Proposition 1. If $B>\bar{\alpha}(\bar{\alpha}+1), u_{M}(p)=\bar{\alpha}$ for all $p \in\left[p_{0} / \bar{\alpha}^{2} ; p_{0}\right]$.

$$
\text { If } B \leq \bar{\alpha}(\bar{\alpha}+1) \text {, }
$$


(i) when $p<p_{0} \frac{1+A}{1+B D}, u_{M}(p)=\frac{p_{0}(1+A)-p(1-B D)}{2 p_{0} A}$,

(ii) when $p \in\left[\frac{p_{0}(1+A)}{1+B D} ; \frac{p_{0}(1+A)}{1+B}\right], u_{M}(p)=\frac{p_{0}(1+A)-p}{p_{0} A}$,

(iii) when $p \in\left[p_{0} \frac{1+A}{1+B} ; p_{0} \frac{\bar{\alpha}(\bar{\alpha}+1)-1}{\bar{\alpha}^{2}(B-1)}\right], u_{M}(p)=\frac{p_{0}(1+A)-p(1-B)}{2 p_{0} A}$,

(iv) when $p>p_{0} \frac{\bar{\alpha}(\bar{\alpha}+1)-1}{\bar{\alpha}^{2}(B-1)}, u_{M}(p)=\bar{\alpha}$.

Proof. From (14), with $\Delta p(t):=p(t+1)-p(t)$, and omitting the $(t)$, we get:

$$
\Delta p=-\left(1-\delta * 1_{\{\Delta p>0\}}\right) \frac{p_{0} A}{B} u+\left(1-\delta * 1_{\{\Delta p>0\}}\right) \frac{1}{B}\left(p_{0}(1+A)-p\right)
$$

and $u$ as a function of $p$ and $\Delta p$ :

$$
u(p, \Delta p)=-\frac{1}{1-\delta * 1_{\{\Delta p>0\}}} \frac{B}{p_{0} A} \Delta p+\frac{1}{p_{0} A}\left(p_{0}(1+A)-p\right) .
$$

Moreover,

$$
\begin{aligned}
K(t+1)-K(t) & =p(t+1) u(t) w-C \\
& =\Delta p(t) u(t) w+p(t) u(t) w-C .
\end{aligned}
$$

Let define $\Delta K(p(t), \Delta p(t)):=K(t+1)-K(t)$ and, let $D:=1 /(1-\delta) \geq 1$. Omitting the $(t)$ again,

$$
\begin{aligned}
\Delta K(p, \Delta p)= & -\frac{B D w}{p_{0} A} \Delta p^{2}+\frac{w}{p_{0} A}\left(p_{0}(1+A)-p(1+B D)\right) \Delta p+\frac{p w}{p_{0} A}\left(p_{0}(1+A)-p\right)-C \\
& \quad \text { if } \Delta p \geq 0 \\
\Delta K(p, \Delta p)= & -\frac{B w}{p_{0} A} \Delta p^{2}+\frac{w}{p_{0} A}\left(p_{0}(1+A)-p(1+B)\right) \Delta p+\frac{p w}{p_{0} A}\left(p_{0}(1+A)-p\right)-C \\
& \text { if } \Delta p \leq 0 .
\end{aligned}
$$

Moreover, when $u$ ranges from 1 to $\bar{\alpha}, \Delta p$ ranges from $\Delta p_{\min }(p)$ to $\Delta p_{\max }(p)$. From (14), the minimum is reached for $u=\bar{\alpha}$, and the maximum for $u=1$, so we have:

$$
\Delta p_{\min }(p)=1 / B\left(p_{0} / \bar{\alpha}^{2}-p\right)
$$

and

$$
\Delta p_{\max }(p)=1 /(B D)\left(p_{0}-p\right) .
$$

So, for a situation described by the pair $(K, p)$, the set of reachable situations at the next time step according to the fraud level chosen by member 
$i, u$, and its anticipation model of other members' behavior (14) is represented in the $(K, p)$-plane by two sections of parabola curves which meet when $\Delta p=0$.

We first consider $\max _{\Delta p \in\left[0 ; \Delta p_{\max }(p)\right]} \Delta K(p, \Delta p)$.

If $p_{0}(1+A)-p(1+B D)>0$, that is $p<p_{0} \frac{1+A}{1+B D}$, the top of the parabola is at $\Delta p^{*}(p):=\frac{p_{0}(1+A)-p(1+B D)}{2 B D}>0$. Moreover, $\Delta p^{*}(p) \leq \Delta p_{\max }(p)$, consequently,

$$
\max _{\Delta p \in\left[0 ; \Delta p_{\max }(p)\right]} \Delta K(p, \Delta p)=\Delta K\left(p, \Delta p^{*}(p)\right) .
$$

If $p_{0}(1+A)-p(1+B D) \leq 0$,

$$
\max _{\Delta p \in\left[0 ; \Delta p_{\max }(p)\right]} \Delta K(p, \Delta p)=\Delta K(p, 0) .
$$

We consider now $\max _{\Delta p \in\left[\Delta p_{\min }(p) ; 0\right]} \Delta K(p, \Delta p)$.

If $p_{0}(1+A)-p(1+B)<0$, that is $p>p_{0} \frac{1+A}{1+B}$, the top of the parabola is at $\Delta p^{* *}(p):=\frac{p_{0}(1+A)-p(1+B)}{2 B}<0$.

Moreover, $\Delta p^{* *}(p) \geq \Delta p_{\min }(p)$, that is $\frac{p_{0}(1+A)-p(1+B)}{2 B} \geq \frac{p_{0} / \bar{\alpha}^{2}-p}{B}$, when $p \leq$ $p_{0} \frac{\bar{\alpha}(\bar{\alpha}+1)-1}{\bar{\alpha}^{2}(B-1)}$ and then :

$$
\max _{\Delta p \in\left[\Delta p_{\min }(p) ; 0\right]} \Delta K(p, \Delta p)=\Delta K\left(p, \Delta p^{* *}(p)\right) .
$$

Otherwise,

$$
\max _{\Delta p \in\left[\Delta p_{\min }(p) ; 0\right]} \Delta K(p, \Delta p)=\Delta K\left(p, \Delta p_{\min }(p)\right) .
$$

If $p_{0}(1+A)-p(1+B) \geq 0$,

$$
\left.\max _{\Delta p \in\left[\Delta p_{\min }(p) ; 0\right]} \Delta K(p, \Delta p)=\Delta K(p, 0)\right) .
$$

Consequently,

(i) when $p<p_{0} \frac{1+A}{1+B D}, \Delta p_{M}(p)=\Delta p^{*}(p)>0$,

(ii) when $p \in\left[\frac{p_{0}(1+A)}{1+B D} ; \frac{p_{0}(1+A)}{1+B}\right], \Delta p_{M}(p)=0$,

(iii) when $\left.p \in] p_{0} \frac{1+A}{1+B} ; p_{0} \frac{\bar{\alpha}(\bar{\alpha}+1)-1}{\bar{\alpha}^{2}(B-1)}\right], \Delta p_{M}(p)=\Delta p^{* *}(p)<0$,

(iv) when $p>p_{0} \frac{\bar{\alpha}(\bar{\alpha}+1)-1}{\bar{\alpha}^{2}(B-1)}, \Delta p_{M}(p)=\Delta p_{\min }(p)<0$. 
Finally, from (19), when $\Delta p_{M}(p)=\Delta p^{*}(p)$, the fraud value, $u_{M}(p)$, that corresponds to the price variation $\Delta p_{M}(p)$ equals

$$
\begin{aligned}
u_{M}(p) & =-\frac{B D}{p_{0} A} \Delta p^{*}(p)+\frac{1}{p_{0} A}\left(p_{0}(1+A)-p\right) \\
& =\frac{1}{2 p_{0} A}\left(p_{0}(1+A)-p(1-B D)\right)
\end{aligned}
$$

when $\Delta p_{M}(p)=\Delta p^{* *}(p)$, the fraud value, $u_{M}(p)$, that corresponds to the price variation $\Delta p_{M}(p)$ equals

$$
\begin{aligned}
u_{M}(p) & =-\frac{B}{p_{0} A} \Delta p^{* *}(p)+\frac{1}{p_{0} A}\left(p_{0}(1+A)-p\right) \\
& =\frac{1}{2 p_{0} A}\left(p_{0}(1+A)-p(1-B)\right),
\end{aligned}
$$

when $\Delta p_{M}(p)=0, u_{M}(p)=\frac{p_{0}(1+A)-p}{p_{0} A}$, and when $\Delta p_{M}(p)=\Delta p_{\min }(p)$, $u_{M}(p)=\bar{\alpha}$.

Corollary 1. Let $(K(n), p(n)), n \in \mathbb{N}$, an evolution governed by the maximal strategy.

- When $B>\bar{\alpha}(\bar{\alpha}+1)$, if $\frac{C}{p_{0} w}>\frac{1}{\bar{\alpha}}$ or

- when $B \leq \bar{\alpha}(\bar{\alpha}+1)$, if $\frac{C}{p_{0} w}>\frac{(1+A)^{2}}{A} \frac{B}{(1+B)^{2}}$

then $\exists N \in \mathbb{N}$ and $\exists c<0$ such that $\forall n \geq N, K(n+1)-K(n)<c$.

Consequently, the evolution $(K(n), p(n))$ is not included in any constraint set (16), the evolutions governed by the maximal strategy are never viable.

Proof. We first show that under the conditions of corollary $1, \Delta K(p, 0)$ is strictly negative when $p \in\left[p_{0} / \bar{\alpha}^{2}, \max \left(p_{0} \frac{1+A}{1+B}, p_{0} / \bar{\alpha}^{2}\right)\right]$.

Let $c(p):=\Delta K(p, 0)=\frac{p w}{p_{0} A}\left(p_{0}(1+A)-p\right)-C$. The top of the parabola is reached for $p=p_{0}(1+A) / 2 \geq \max \left(p_{0} \frac{1+A}{1+B}, p_{0} / \bar{\alpha}^{2}\right)$, so $c$ is increasing between $p_{0} / \bar{\alpha}^{2}$ and $\max \left(p_{0} \frac{1+A}{1+B}, p_{0} / \bar{\alpha}^{2}\right)$.

Consequently, $\forall p \in\left[p_{0} / \bar{\alpha}^{2}, \max \left(p_{0} \frac{1+A}{1+B}, p_{0} / \bar{\alpha}^{2}\right)\right], c(p) \leq c\left(\max \left(p_{0} \frac{1+A}{1+B}, p_{0} / \bar{\alpha}^{2}\right)\right)$.

- when $B>\bar{\alpha}(\bar{\alpha}+1)$, we have $p_{0} / \bar{\alpha}^{2}>p_{0} \frac{1+A}{1+B}$.

Since $c\left(p_{0} / \bar{\alpha}^{2}\right)=\frac{w p_{0}}{\bar{\alpha}}-C$, if $\frac{w p_{0}}{\bar{\alpha}}-C<0, c\left(\max \left(p_{0} \frac{1+A}{1+B}, p_{0} / \bar{\alpha}^{2}\right)\right)<0$ and

$$
\forall p \in\left[p_{0} / \bar{\alpha}^{2} ; \max \left(p_{0} \frac{1+A}{1+B}, p_{0} / \bar{\alpha}^{2}\right)\right], \Delta K(p, 0)=c(p)<0 .
$$


- when $B \leq \bar{\alpha}(\bar{\alpha}+1)$, we have $p_{0} / \bar{\alpha}^{2} \leq p_{0} \frac{1+A}{1+B}$.

Since $c\left(p_{0} \frac{1+A}{1+B}\right)=\frac{(1+A)^{2} w p_{0}}{A} \frac{B}{(1+B)^{2}}-C$, if $\frac{(1+A)^{2} w p_{0}}{A} \frac{B}{(1+B)^{2}}-C<0$, $c\left(\max \left(p_{0} \frac{1+A}{1+B}, p_{0} / \bar{\alpha}^{2}\right)\right)<0$ and

$$
\forall p \in\left[p_{0} / \bar{\alpha}^{2} ; \max \left(p_{0} \frac{1+A}{1+B}, p_{0} / \bar{\alpha}^{2}\right)\right], \Delta K(p, 0)=c(p)<0 .
$$

Now, let $(K(n), p(n))$ an evolution governed by the maximal strategy. Necessarily, $p(0) \in\left[p_{0} / \bar{\alpha}^{2} ; p_{0}\right]$. Moreover, from (29),

- if $p(0)>p_{0} \frac{1+A}{1+B}$, then $p(n)$ tends toward $\max \left(p_{0} \frac{1+A}{1+B}, p_{0} / \bar{\alpha}^{2}\right)$,

- if $p_{0} \frac{1+A}{1+B D} \leq p(0) \leq p_{0} \frac{1+A}{1+B}$, then for all $n, p(n)=p(0)$,

- if $p(0)<p_{0} \frac{1+A}{1+B D}$, then $p(n)$ tends toward $p_{0} \frac{1+A}{1+B D}$.

Thus, for all $p(0) \in\left[p_{0} / \bar{\alpha}^{2} ; p_{0}\right], \lim _{n} p(n)=\hat{p} \in\left[p_{0} / \bar{\alpha}^{2} ; \max \left(p_{0} / \bar{\alpha}^{2}, p_{0}(1+\right.\right.$ $A) /(1+B))]$ and $\lim _{n} p(n+1)-p(n)=0$.

Consequently, the capital variation $K(n+1)-K(n)$ tends toward $\Delta K(\hat{p}, 0)=$ $c(\hat{p})$ which is strictly negative from $(32)$ and (33) since $\hat{p} \in\left[p_{0} / \bar{\alpha}^{2} ; \max \left(p_{0} / \bar{\alpha}^{2}, p_{0}(1+\right.\right.$ $A) /(1+B))]$. Consequently, the evolution $(K(n), p(n))$ is not viable.

Figures $1(\mathrm{a})$ et $1(\mathrm{~b})$ display $u_{M}$ and $\Delta p_{M}$ according to $p$ with parameter values such that according to Corollary 1, the maximal strategy is never viable. Figure 1(a) shows that such a strategy implies high fraud values for all $p$. Moreover, figure $1(\mathrm{~b})$ shows that necessarily such a strategy converges toward a fraud level associated with a negative value of $\Delta K$ and so leads to ruin.

\subsection{The maximal viable strategy}

The maximal viable individual benefit strategy consists in maximizing at each time step the agent benefit while ensuring the constraint satisfaction over time.

From [8], given a control dynamical system

$$
\begin{cases}x(t+1) & =f(x(t), u(t)) \\ u(t) & \in U(x(t))\end{cases}
$$




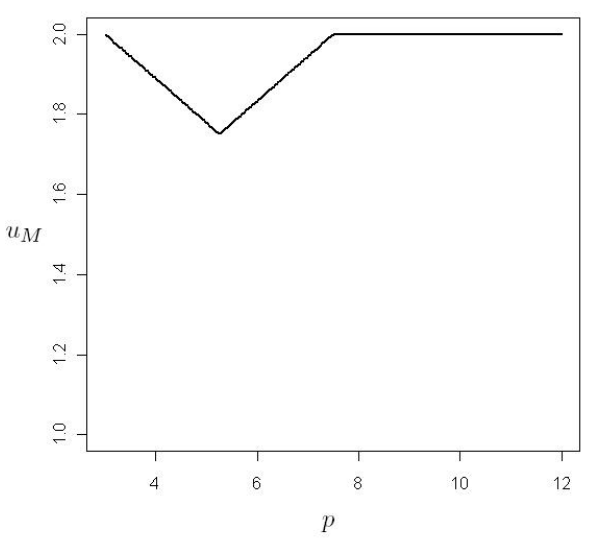

(a)

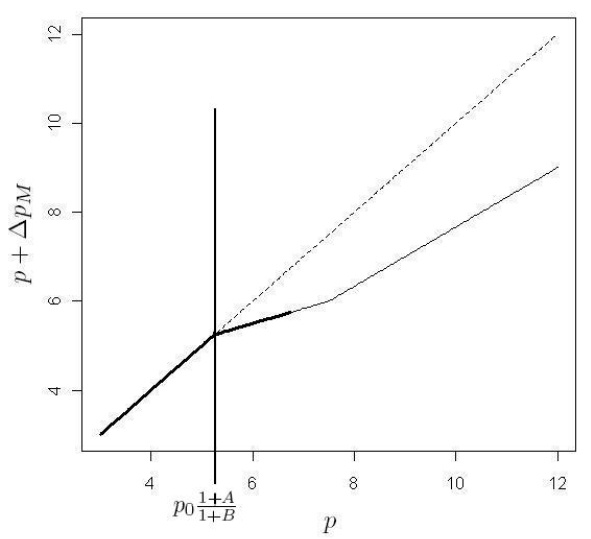

(b)

Figure 1: The parameter values are $p_{0}=12, \bar{\alpha}=2, w=1, C=11, B=3$ and $D=$ 2. Figure 1(a) displays $u_{M}$ according to $p$. With these values $p_{0} \frac{1+A}{1+B}=5.25$. Figure 1 (b) displays $p+\Delta p_{M}(p)$ according to $p$ with bold line when $\Delta K\left(p, \Delta p_{M}(p)\right)<0$.

and a constraint set, $L$, the first step to determine viable strategies is to compute the viability kernel that is the subset of $L$ gathering all states of the system such that there exists at least one control function that allows the system to remain in the constraint set indefinitely ${ }^{1}$ :

$$
\left.\operatorname{Viab}_{(34)}(L)=\{x \in L|\exists u(.)| x(t) \in L \forall t \in \mathbb{N}\}\right) .
$$

The states belonging to the viability kernel are called viable states and the control functions that allow to remain in the viability kernel are called viable control functions. Such functions can be derived from viability kernel determination: These are the control functions such that system state remains in the viability kernel at next time step.

Consequently, the computation of the viability kernel of the local viability problem of one cooperative member, $\operatorname{Viab}_{(14)}(L) \subset R^{2}$, associated with the control dynamical system (14) and the constraint set (15) is a preliminary to maximal viable strategy determination. Actually, viable strategies can only be defined for pairs $(K, p)$ belonging to the viability kernel. Outside,

\footnotetext{
${ }^{1}$ In the discrete time case, the assumptions are that the control dynamical system has to be Lipschitz with closed images and the constraint set has to be closed.
} 
constraints will be violated whatever the fraud strategy. In particular, if the viability kernel is empty, from any point, there is no viable strategy. The following theorem specifies necessary and sufficient conditions for the viability kernel to be non empty.

Theorem 1. Let $f:] 0 ; 2] \rightarrow \mathbb{R}$ such that $f(A)=\frac{(1+A)^{2}}{4 A}$ if $A \leq 1$ and $f(A)=$ 1 otherwise.

$\operatorname{Viab}_{(14)}(L) \neq \emptyset$ if and only if $\frac{C}{p_{0} w} \leq f(A)$.

Proof. We give here a sketch of the proof, the complete proof is in Appendix A.

When the condition on the parameter values of Theorem 1 is satisfied, we can exhibit a viable evolution so the viability kernel is not empty.

When the condition on the parameter values of Theorem 1 is not satisfied, the third term of the quadratic function describing $\Delta K(p,$.$) according to \Delta p$ is strictly negative whatever the value of $p(21)$. The first consequence is that if $\Delta p=0, \Delta K(p, 0)<0$. The first part of the proof consists in comparing the marginal capital growth associated with an increase of the price with the marginal capital growth associated with a decrease of the price. This comparison allows to show in the second part of the proof that any capital evolution is lower than a sequence which tends toward $-\infty$.

We compute the viability kernels following the algorithm of [35]. Since the variations of the state variables $(K, p)$ do not depend on $K$, the results for $K_{\min }>0$ can be deduced from those with $K_{\min }=0$ by a translation of $+K_{\min }$ on the $K$-axis. We then set $K_{\min }=0$ in the following, the constraint to be satisfied is then $K \geq 0$.

Given a maximal exploration value for $K, K_{\max }$, given discretization steps for $p$ and $K$, all pairs $(K, p) \in\left[0 ; K_{\max }\right] \times\left[p_{0} / \bar{\alpha}^{2} ; p_{0}\right]$ belonging to the discretization grid constitute the set to be tested at the initialization of the algorithm. At each iteration of the algorithm, pairs are removed if they have no successor by (14) in the current set to be tested. Algorithm stops when no pair is removed during an iteration.

Figure 2 displays the viability kernel for dynamics (14) and constraint set (15) for different values of $C$ and for different values of $B$. The viability kernel volume decreases with $C$ and becomes empty when $\frac{C}{p_{0} w}>\frac{(1+A)^{2}}{4 A}$ as stated in Theorem 1 (Fig. 2(a)). It decreases with $B$ (Fig. 2(b)) since the effect of agent $i$ 's fraud on the global quality is inversely proportional to $B$. 


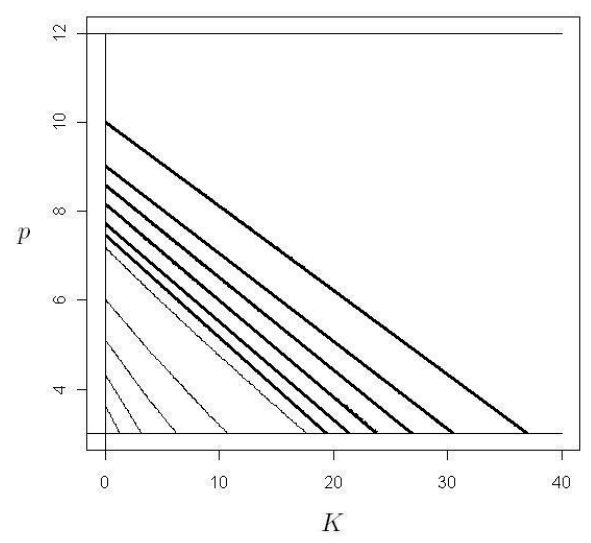

(a)

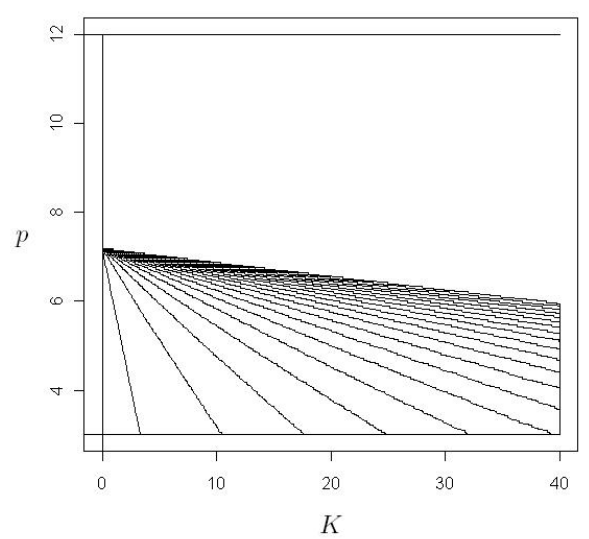

(b)

Figure 2: The parameter values are $p_{0}=12, \bar{\alpha}=2, w=1, D=2$, so $\frac{p_{0} w(1+A)^{2}}{4 A}=$ 12.25. Figure 2 (a) displays the lower boundary of the viability kernel for $B=3$ and $C=7,8,9,10,11$ for the plain lines and $C=11.2,11.4,11.6,11.8,12,12.2$ for the bold lines. The viability kernel volume decreases with $C$. Figure 2(b) displays the lower boundary of the viability kernel for $C=11$ and $B=2,3, \ldots, 40$. The viability kernel volume decreases with $B$.

Let $\Delta p_{M V}(K, p)$ and $u_{M V}(K, p)$ the values of the global price variation and the values of the fraud levels of member $i$ associated with the maximal viable benefit strategy, these functions are only defines on $\operatorname{Viab}_{(14)}(L)$ and contrary to the maximal strategy, these values depend on $K$ :

$$
\Delta p_{M V}(K, p)=\underset{\substack{\Delta p \in\left[\Delta p_{\min }(p) ; \Delta p_{\max }(p)\right] \\(K+\Delta K(p, \Delta p), p+\Delta p) \in \operatorname{Viab}_{(14)}(L)}}{\operatorname{argmax}} \Delta K(p, \Delta p)
$$

From (19),

$$
\begin{aligned}
u_{M V}(K, p)= & -\frac{B D}{p_{0} A} \Delta p_{M V}(K, p)+\frac{1}{p_{0} A}\left(p_{0}(1+A)-p\right) \\
& \text { if } \Delta p_{M V}(K, p) \geq 0 \\
u_{M V}(K, p)= & -\frac{B}{p_{0} A} \Delta p_{M V}(K, p)+\frac{1}{p_{0} A}\left(p_{0}(1+A)-p\right) \\
& \text { if } \Delta p_{M V}(K, p)<0 .
\end{aligned}
$$

The values of $u_{M V}$ according to $(K, p)$ are displayed in figure 3(a). Figures 3(a) and 3(b) show that when $K$ is high, maximal and maximal viable 
strategies merge: Constraint set boundary $K=0$ is too far to have an influence. Figure 3(a) shows that for smaller $K$ values, halting fraud is necessary to avoid ruin.

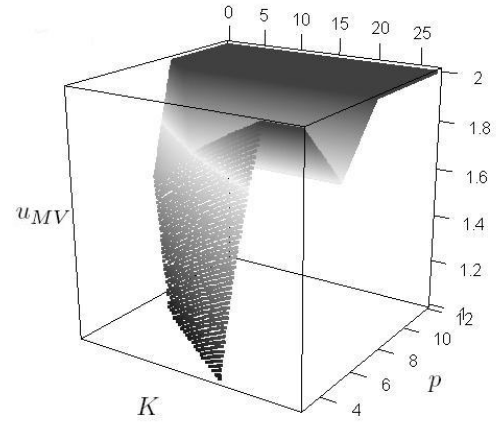

(a)

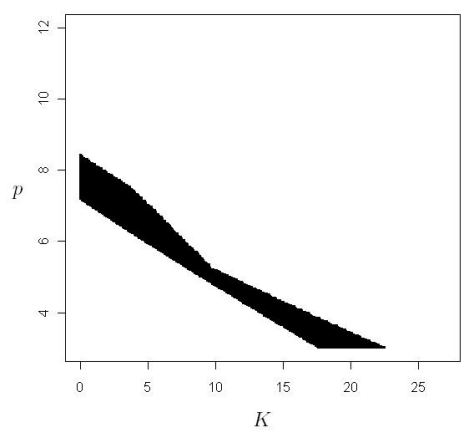

(b)

Figure 3: The parameter values are $p_{0}=12, \bar{\alpha}=2, w=1, D=2, B=3$ and $C=11$. Figure $3(\mathrm{a})$ displays $u_{M V}$ according to $(K, p)$. In figure $3(\mathrm{~b})$, the area where $u_{M V}(K, p)$ differs from $u_{M}(p)$ is colored black.

Figure 4(a) gives the capital variation values, $\Delta K\left(p, \Delta p_{M V}(K, p)\right)$, associated with the maximal viable strategy according to $(K, p)$. In the area where the maximal and the maximal viable strategy differ, the capital variation obviously also differs. Figure 4(b) shows the capital lost during one time step by pro-viable agent compared to a maximal one according to $K$ and $p, \Delta K\left(p, \Delta p_{M V}(K, p)\right)-\Delta K\left(p, \Delta p_{M}(p)\right)$.

\section{Practical issue: Can local strategies of pro-viable agents ensure collective viability?}

In the previous sections we have studied the local behavior of the agents, when they work out their strategy with their own model of the cooperative. We now study the real viability of the combination of the local strategies of the members of the cooperative.

\subsection{Definition of the viability of the cooperative}

The viability of the cooperative depends on the number of agents that remains in the cooperative. $N(t)$ is the state variable of the cooperative on which the viability constraint is defined. 


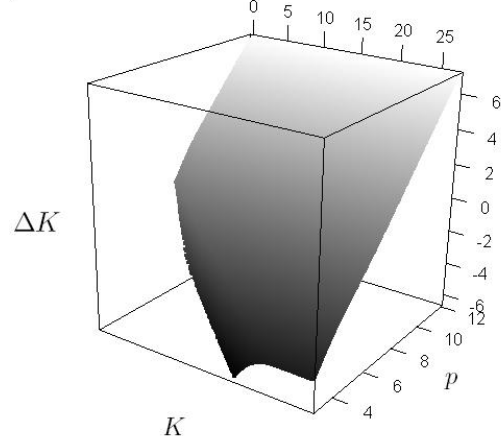

(a)

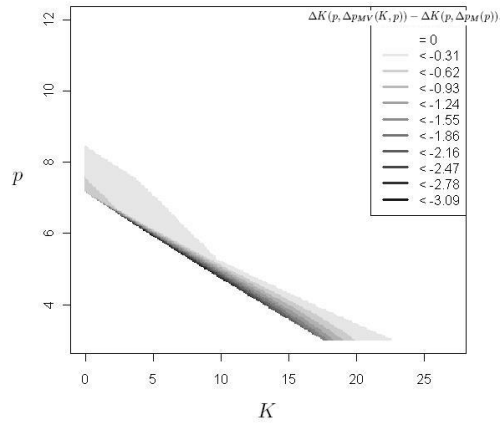

(b)

Figure 4: The parameter values are $p_{0}=12, \bar{\alpha}=2, w=1, D=2, B=3$ and $C=$ 11. Figure 4(a) displays capital variations according to $(K, p)$ when $u=u_{M V}$. In figure $4(\mathrm{~b})$, the area where $u_{M V}(K, p)$ differs from $u_{M}(p)$ is colored according to the difference between the capital variations associated with $u=u_{M}$ and the capital variations associated with $u=u_{M V}, \Delta K\left(p, \Delta p_{M V}(K, p)\right)-\Delta K\left(p, \Delta p_{M}(p)\right)$.

Definition 1. A cooperative is strongly viable when $N(t)=N(0), \forall t \in \mathbb{N}$.

Definition 2. A cooperative is weakly viable when $N(t) \geq N_{\min }, \forall t \in \mathbb{N}$.

We consider here that agents leave the cooperative when their capital $K_{i}$ becomes too low $\left(K_{i}<K_{i_{m i n}}\right)$, and we suppose that no new agent enters the cooperative.

\subsection{Cooperative of Myopic agents}

When all members of the cooperative are myopic agents, the price remains constant, as all agents' capital variation and fraud level. From (19), an agent $i$ is viable (according to the local definition) if:

$$
K_{i}(1)-K_{i}(0)=\frac{p(0) w_{i}\left(p_{0}(1+A)-p(0)\right)}{p_{0} A}-C_{i} \geq 0
$$

since $\forall t, K_{i}(t+1)-K_{i}(t)=K_{i}(1)-K_{i}(0)$. Consequently the cooperative is strongly viable if all agents remain in the cooperative, that is when:

$$
\forall i, \quad \frac{p(0) w_{i}\left(p_{0}(1+A)-p(0)\right)}{p_{0} A}-C_{i} \geq 0 .
$$


Let $M:=\operatorname{card}\left\{i / \Delta K_{i}(0) \geq 0\right\}$, then the cooperative is weakly viable for $N_{\min } \leq M$. In all other cases, the cooperative is not viable.

Proposition 2. Let $f:] 0 ; 2] \rightarrow \mathbb{R}$ such that $f(A)=\frac{(1+A)^{2}}{4 A}$ if $A \leq 1$ and $f(A)=1$ otherwise (as in theorem 1).

When all members of the cooperative are myopic agents, a myopic agent is never viable whatever the initial price value when $\frac{C_{i}}{w_{i} p_{0}}>f(A)$.

When $\frac{C_{i}}{w_{i} p_{0}} \leq f(A)$, this myopic agent is viable if and only if

$p(0) \in\left[p_{Z_{\text {imin }}} ; p_{Z_{\text {imax }}}\right]:=\left[\max \left(p_{0} / \bar{\alpha}^{2}, \frac{p_{0}(1+A)-\sqrt{\hat{\Delta}}}{2}\right) ; \min \left(\frac{p_{0}(1+A)+\sqrt{\hat{\Delta}}}{2}, p_{0}\right)\right]$

where

$$
\hat{\Delta}:=p_{0}^{2}(1+A)^{2}-4 \frac{p_{0} A C_{i}}{w_{i}} .
$$

Proof. Proposition 2 is a consequence of the study of the quadratic equation (37).

We note $Z_{i}$ the area of the state space $(K, p)$ delimited by the values of $\left(K_{i}(0), p(0)\right)$ for which myopic agent $i$ is viable in a cooperative of myopic agents (see figure 5),

$$
Z_{i}=\left[0 ;+\infty\left[\times\left[p_{Z_{\text {imin }}} ; p_{Z_{\text {imax }}}\right]\right.\right.
$$

with $p_{Z_{i m i n}}$ and $p_{Z_{\text {imax }}}$ defined in (39).

\subsection{Mixture of agents}

For sake of simplicity, we assume from here on that all agents have the same production quantity and charge:

$$
\forall i, w_{i}=w \text { and } C_{i}=C \text {. }
$$

In that case, $B_{i}$ can be interpreted as the inverse of the proportion of cooperative members that act like agent $i$. Let $k_{i}$ such that $B_{i}=N / k_{i}$, this means that agent $i$ assumes that:

- $k_{i}$ members among $N$ will make the same fraud level choice as itself;

- the $N-k_{i}$ other members are myopic agents (they choose on average a next fraud level equal to the previous one). 


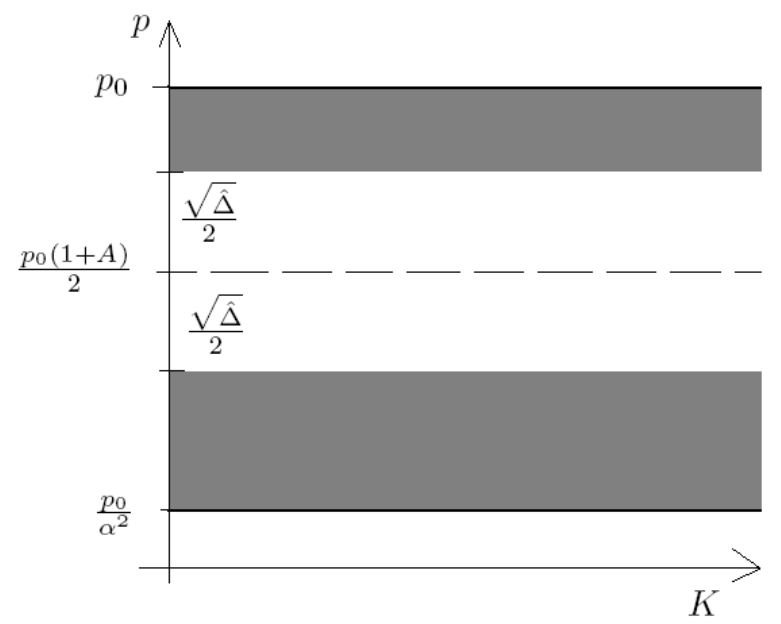

Figure 5: The area $Z_{i}$ is colored white: If $\left(K_{i}(0), p(0)\right) \in Z_{i}$, then the myopic agent $i$ is viable in a cooperative of myopic agents.

\subsubsection{Mixture of myopic and maximizing agents}

We consider now a mixture of $k$ maximizing agents and $N-k$ myopic agents. We assume that the maximizing agents have an accurate perception of the other members' behavior, that is that their parameter $B_{i}=B=N / k$. The following proposition is a consequence of corollary 1 :

Proposition 3. Let a mixture of myopic and maximizing agents satisfying condition (41).

- When $B>\bar{\alpha}(\bar{\alpha}+1)$, if $\frac{C}{p_{0} w}>\frac{1}{\bar{\alpha}}$ or

- when $B \leq \bar{\alpha}(\bar{\alpha}+1)$, if $\frac{C}{p_{0} w}>\frac{(1+A)^{2}}{A} \frac{B}{(1+B)^{2}}$

then neither maximizing nor myopic agents of the mixture are viable.

From the previous subsection we know that there are values of $\left(K_{i}(0), p(0)\right)$ for which myopic agents alone are viable when $\frac{C}{w p_{0}} \leq f(A)$.

Incorporating maximizing agents provoke then a complete loss of viability (myopic agents alone are viable for $\left(K_{i}(0), p(0)\right) \in Z$, whereas all the agents of the mixture are never viable whatever the value of $\left.\left(K_{i}(0), p(0)\right)\right)$ when:

$$
\frac{C}{w p_{0}} \in\left[\frac{1}{\bar{\alpha}} ; f(A)\right] \text { with } B>\bar{\alpha}(\bar{\alpha}+1),
$$


or

$$
\frac{C}{w p_{0}} \in\left[\frac{(1+A)^{2}}{A} \frac{B}{(1+B)^{2}}, f(A)\right] \text { with } B \leq \bar{\alpha}(\bar{\alpha}+1) .
$$

Figure 6 illustrates this loss of global viability: the area colored in grey represents the values of the initial price for which a cooperative of myopic agents is viable according to the value of $\frac{C}{p_{0} w}$. When maximizing agents are included in the mixture, the area of initial price for which the mixture is viable is reduced to the left side of the vertical lines; their positions depend on the proportion of maximazing agents described by parameter $B$.

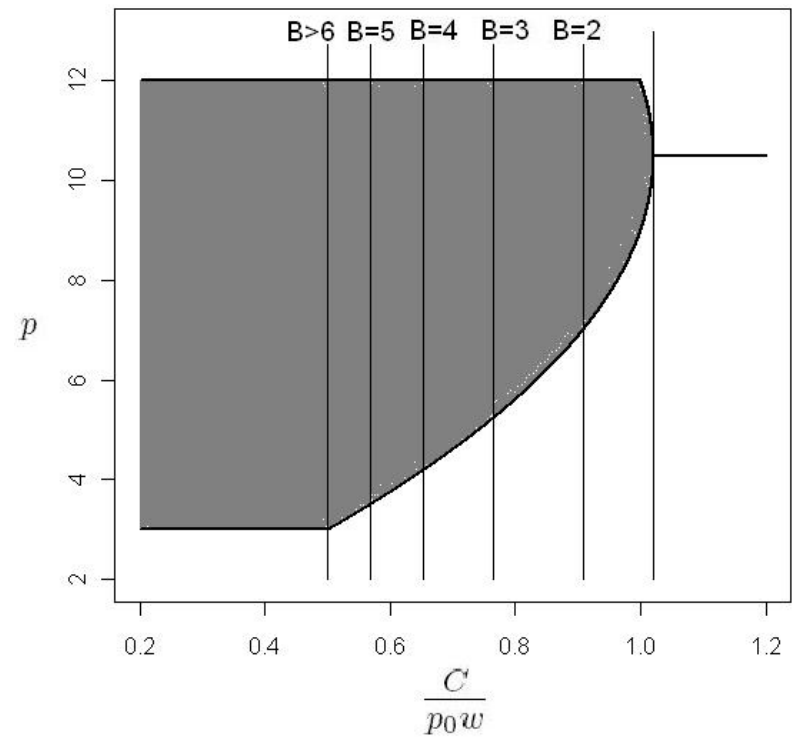

Figure 6: The upper curve represents $p_{Z_{\max }}$ according to $\frac{C}{p_{0} w}$. The lower curve represents $p_{Z_{\text {min }}}$ according to $\frac{C}{p_{0} w}$. The area which is colored grey represents the values of the initial price for which a cooperative of myopic agents is viable. When maximizing agents are included in the mixture, the area of initial price for which the mixture is viable is reduced to the left side of the vertical lines. $p_{0}=12, \bar{\alpha}=2$ and from (43), the position of the vertical lines is defined by $\frac{C}{p_{0} w}=\frac{(1+A)^{2}}{A} \frac{B}{(1+B)^{2}}$ when $B \leq \bar{\alpha}(\bar{\alpha}+1)=6$ and by $\frac{C}{p_{0} w}=\frac{1}{\bar{\alpha}}$ when $B>\bar{\alpha}(\bar{\alpha}+1)=6$.

\subsubsection{Mixture of myopic and pro-viable agents}

Theorems on pro-viable agents individual viability cannot help to derive any conclusion on the viability of a mixture of myopic and pro-viable agents. It is necessary to study the viability of the mixture through simulation.

However, 
Lemma 1. With condition (41), the area $Z$ for which the myopic agents are viable is included in the viability kernel of each pro-viable agent.

Proof. Let $(K, p) \in Z, K \geq K_{\text {min }}$ and $c(p) \geq 0$. So, $\Delta K(p, 0)=c(p) \geq 0$ and the evolution such that $(K(0)=K, p(0)=p)$ and for all $t \in \mathbb{N}, \Delta p(t)=0$ is viable.

To highlight the influence of the pro-viable agents on the collective viability, we now study the behavior of a mixture of myopic and pro-viable agents in a parameter range such that myopic agents alone can be viable (depending on the initial value of $p$ ) and such that no mixture with maximizing agents is ever viable. From the preceding subsection, we choose then simulation parameters which satisfy conditions (42) or (43).

In particular, we have chosen:

- $p_{0}=12$

- $\bar{\alpha}=2$, and therefore $A=\frac{3}{4}$

- $w=1$

- $C=11$

- $\delta=0.5$

- $K_{\min }=0$ for the pro-viable agents

- $K(0)=20$ is the same for all pro-viable agents

- $N=24$ (similar results are obtained with other values for $N$ )

- $k=4(B=6), k=6(B=4), k=8(B=3), k=10(B=2.4)$.

With these parameters, the conditions expressed in (43) are verified and we have $p_{Z_{\text {min }}}=\frac{21-3 \sqrt{5}}{2}(39)$.

As in the mixture with myopic and maximizing agents, we assume that pro-viable agents have an accurate perception of the other members' behavior $\left(B_{i}=B=N / k\right)$.

All simulation results indicate that: 


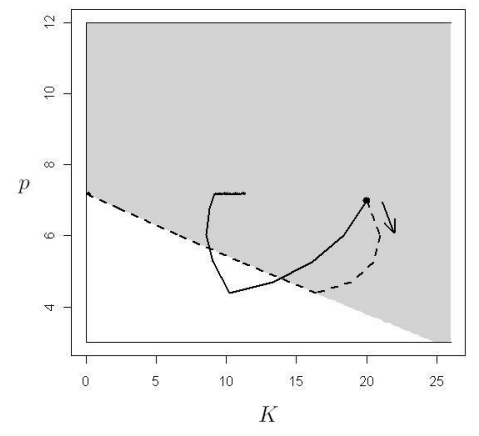

(a)

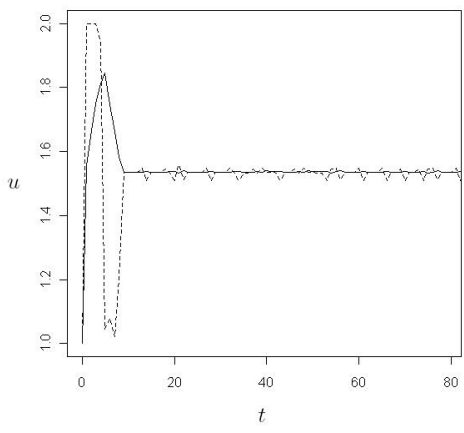

(c)

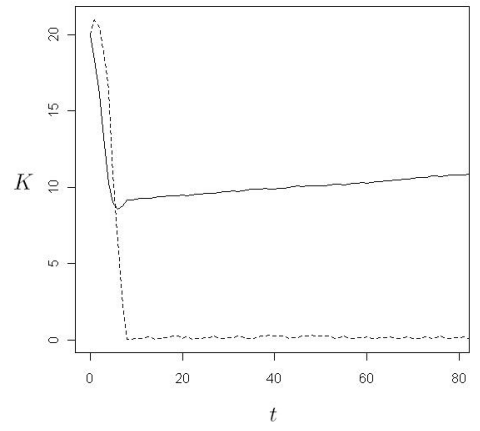

(b)

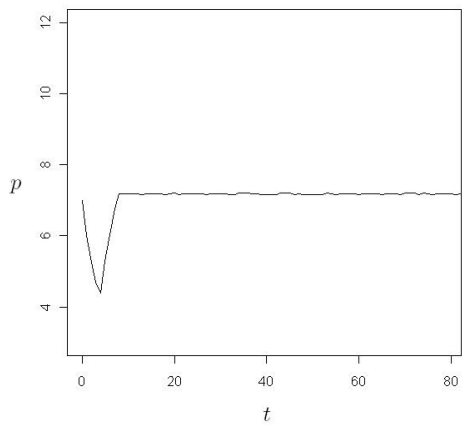

(d)

Figure 7: The parameter values are $N=24, k=6(B=4), K(0)=20$ and $p(0)=7$. Figure 7(a) displays the trajectory of the evolution of the global price and the capital of the pro-viable agents (dotted line) and of the global price and the capital of the myopic agents (plain line) in the $(K, p)$-plane. The viability kernel of the pro-viable agents is colored gray. Figure 7(b) show the evolution of the capital of the pro-viable agents (dotted line) and of the capital of the myopic agents (plain line) according to time. Figure 7(c) show the evolution of the fraud level of the pro-viable agents (dotted line) and of the fraud level of the myopic agents (plain line) according to time. Figure 7(d) show the evolution of the global price according to time.

- Pro-viable agents begin with increasing their capital when it is possible (at least when $p(t) \geq p_{Z_{m i n}}$ but not only, see figure 9 ). This is achieved by increasing their fraud level, therefore $p(t)$ decreases as does the myopics' capital (since their fraud level is always lower than the pro- 


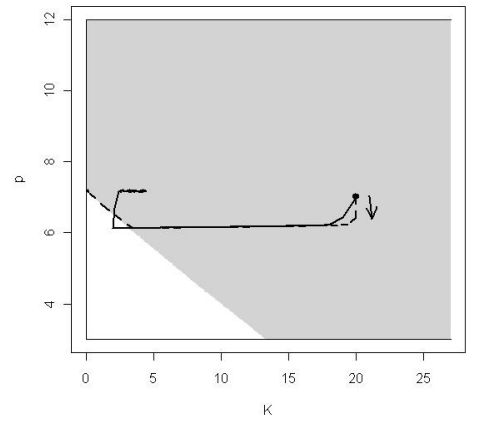

(a)

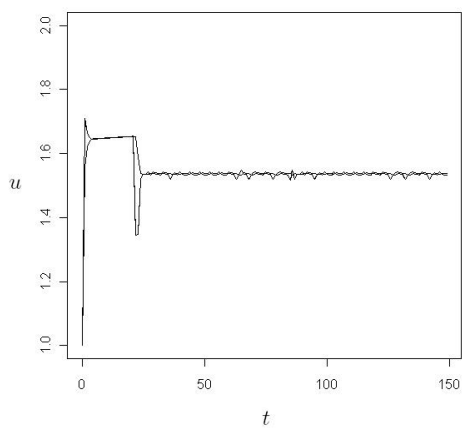

(c)

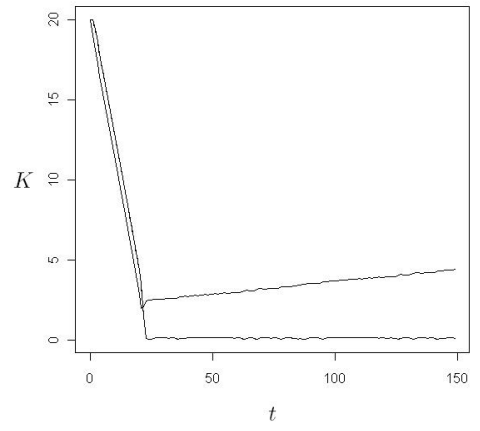

(b)

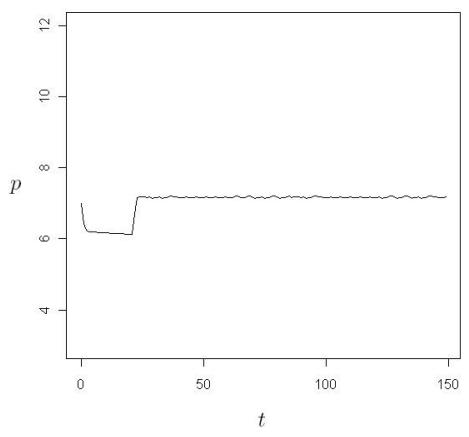

(d)

Figure 8: The parameter values are $N=24, k=10(B=2.4), K(0)=20$ and $p(0)=7$. Figure 8(a) displays the trajectory of the evolution of the global price and the capital of the pro-viable agents (dotted line) and of the global price and the capital of the myopic agents (plain line) in the $(K, p)$-plane. The viability kernel of the pro-viable agents is colored gray. Figure 8(b) show the evolution of the capital of the pro-viable agents (dotted line) and of the capital of the myopic agents (plain line) according to time. Figure 8(c) show the evolution of the fraud level of the pro-viable agents (dotted line) and of the fraud level of the myopic agents (plain line) according to time. Figure 8(d) show the evolution of the global price according to time.

viable agents fraud level at this stage) (Fig. 7(a), 8(a), 9(a) and 10(a)).

- When $p(t)$ becomes too low, pro-viable agents behave as maximizing agents as long as they are not near the boundary of the viability kernel: They keep an almost constant fraud level, therefore $p(t)$ remains 


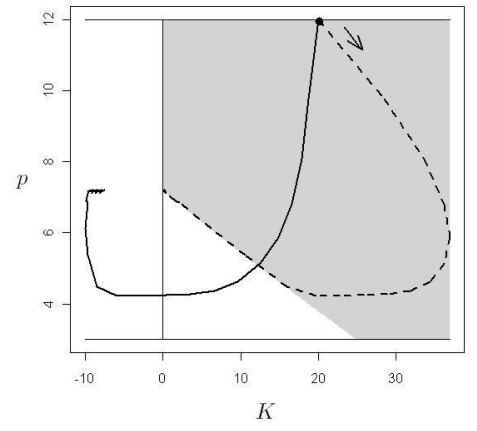

(a)

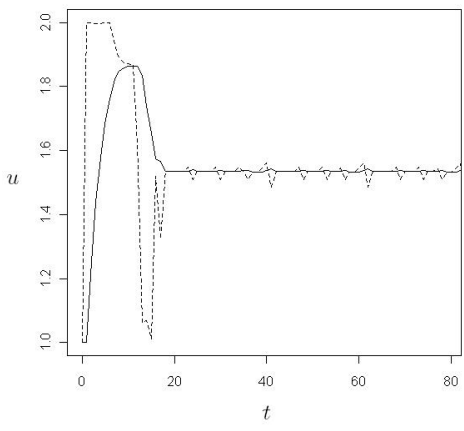

(c)

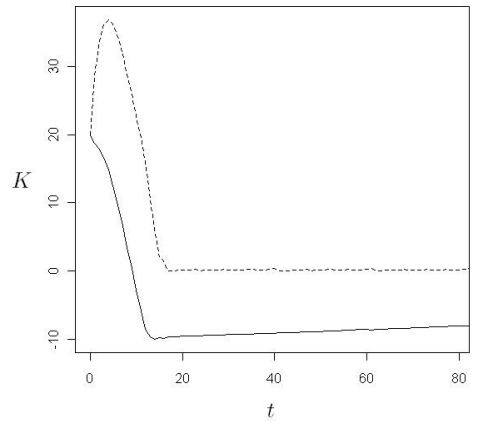

(b)

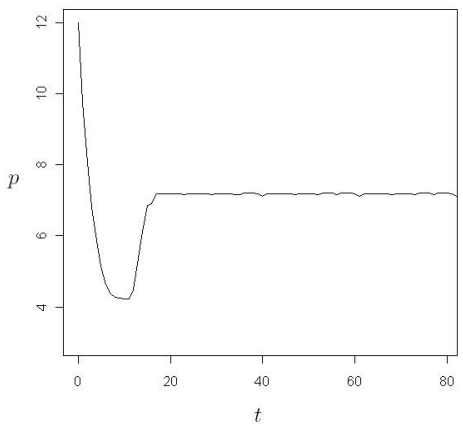

(d)

Figure 9: The parameter values are $N=24, k=6(B=4), K(0)=20$ and $p(0)=12$. Figure 9(a) displays the trajectory of the evolution of the global price and the capital of the pro-viable agents (dotted line) and of the global price and the capital of the myopic agents (plain line) in the $(K, p)$-plane. The viability kernel of the pro-viable agents is colored gray. Figure 9(b) show the evolution of the capital of the pro-viable agents (dotted line) and of the capital of the myopic agents (plain line) according to time. Figure 9(c) show the evolution of the fraud level of the pro-viable agents (dotted line) and of the fraud level of the myopic agents (plain line) according to time. Figure 9(d) show the evolution of the global price according to time.

constant (Fig. $7($ d) , 8(d), 9(d) and 10(d)) but all agents loose money (Fig. 7(b), 8(b), 9(b) and 10(b)).

- When the pro-viable agents reach the boundary of their viability kernel, they change radically their behavior (Fig. 7(a), 8(a), 9(a) and 


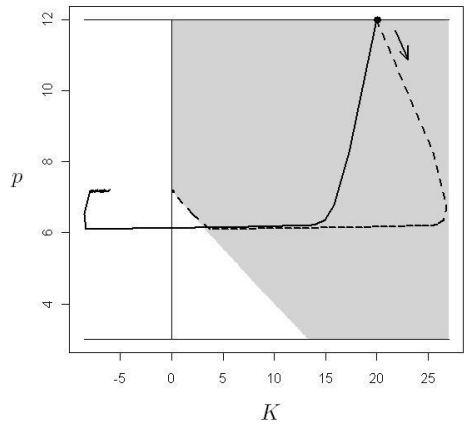

(a)

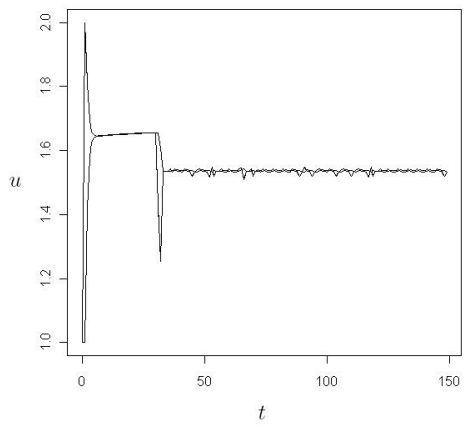

(c)

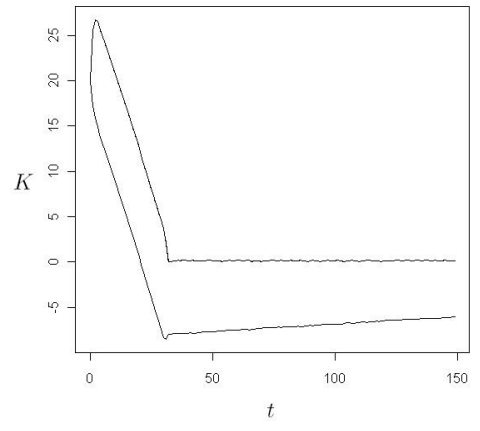

(b)

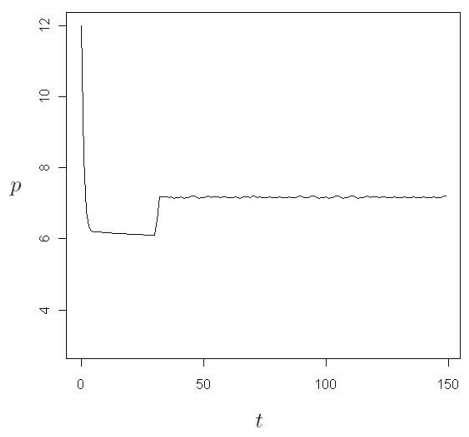

(d)

Figure 10: The parameter values are $N=24, k=10(B=2.4), K(0)=20$ and $p(0)=12$. Figure 10(a) displays the trajectory of the evolution of the global price and the capital of the pro-viable agents (dotted line) and of the global price and the capital of the myopic agents (plain line) in the $(K, p)$-plane. The viability kernel of the pro-viable agents is colored gray. Figure 10(b) show the evolution of the capital of the pro-viable agents (dotted line) and of the capital of the myopic agents (plain line) according to time. Figure 10(c) show the evolution of the fraud level of the pro-viable agents (dotted line) and of the fraud level of the myopic agents (plain line) according to time. Figure 10(d) show the evolution of the global price according to time.

$10(\mathrm{a}))$. They reduce their fraud level until $p(t)$ becomes close to $p_{Z_{\min }}$ (Fig. $7(\mathrm{~d}), 8(\mathrm{~d}), 9(\mathrm{~d})$ and $10(\mathrm{~d}))$. In this phase, myopic agents loose less money than the pro-viable ones. Pro-viable agents evolve on the boundary of the viability kernel until they reach the constraint bound- 
ary $K=0$. After that their fraud level stays very close to $u\left(p_{Z_{\text {min }}}\right)(19)$ (Fig. 7(c), 8(c), 9(c) and 10(c)). Myopic agents no longer loose money.

- Myopic agents' capital has a lower bound, which can be negative, depending on $p(0), K(0)$, and $\frac{k}{N}$ (Fig. $7(\mathrm{~b}), 8(\mathrm{~b}), 9(\mathrm{~b})$ and $10(\mathrm{~b})$ ).

The experiment suggests that when $p(0)$ and $K(0)$ are chosen inside the viability kernel of pro-viable agents, then the myopic agents' capital has always a lower bound. This means that pro-viable agents increase the viability area of myopic agents. The viable area gained by myopic agents depends on the proportion of pro-viable agents, as shown in Figure 2(b). With one single pro-viable agent here $B=24$ the area gained is rather small, but with $B=2.4$ and $K(0)=20$ it is possible to choose as initial value of price the lowest price $p(0)=\frac{p_{0}}{\bar{\alpha}^{2}}$.

We then conjecture that the cooperative is strongly viable as soon as the myopic agents $K_{\min }$ is lower than this lower bound.

Figures 7 and 8 show how the cooperative agents evolve for different proportions of pro-viable agents (respectively $1 / 4$ and 5/12, other parameters give similar results and figures). These are cases where myopic agents alone are not viable $(p(0)=7$ does not belong to $Z)$. In all cases the cooperative becomes viable (since $(p(0)=7, K(0)=20)$ is inside the local viability kernel of pro-viable agents). However the minimum capital of myopic agents is lower as the proportion of pro-viable agents increases as shown in Table 1. When there are only pro-viable agents left, their minimum capital is 0 .

$\begin{array}{cccc}N & k & \min _{t}\{K(t)\} \text { for all myopic agents } & \min _{t}\{K(t)\} \\ & & \text { for all myopic agents } \\ 24 & 4 & 10.83 & \text { when } p(0)=12 \\ 24 & 6 & 8.56 & -17.17 \\ 24 & 8 & 4.46 & -10.05 \\ 24 & 10 & 2.00 & -9.06 \\ & \end{array}$

Table 1: Minimal capital value over time, $\min _{t}\{K(t)\}$, for all myopic agents depending on the proportion of pro-viable agents.

When $p(0) \in Z$, for example $p(0)=12$, myopic agents are viable by themselves (their income is positive at each time step). In a mixture with proviable agents their capital can be lower than when they are by themselves. It can even be negative. Figures 9 and 10 show for different proportions 
of pro-viable agents (respectively $1 / 4$ and 5/12) how myopic agents capital first decreases to a minimum and then increases slightly (other values for $k / N$ satisfying condition (43) give similar shapes). The minimum capital of myopic agents is negative but increases with the proportion of pro-viable agents as shown in Table 1.

\section{Conclusion}

We have studied a non classical viability problem which encompasses $N$ linked local viability problems and which is then in general non tractable by the existing viability algorithms due to the high dimensionality. We consider the case of a stylized agricultural cooperative. The cooperative members income is a function of their economic ratio $\lambda=\frac{C}{w p_{0}}$, their fraud level (between 1 and $\bar{\alpha}$ ) and the price which depends on the average fraud level. Three kinds of strategies are taken into account: Myopic agents (which imitate the average behavior), maximizing agents and pro-viable agents. These last two types of agents use a model of the other's behavior characterized by the proportion $(1 / B)$ of agents acting like themselves.

We have proved that the strategy of maximizing agents does not always correspond to maximal fraud, and we have identified the range of values of parameters for which this behavior prevails. In particular, this happens when the proportion of maximizing agents is high and the price is not too high. We have also proved that for some range of values of the proportion $1 / B$ and of the micro-economic ratio $\lambda=\frac{C}{w p_{0}}$, the maximizing strategy is never viable.

Regarding pro-viable agents, which maximize their income while ensuring their own viability, we have proved that the local viability kernel is not empty if and only if the micro-economic ratio $\lambda$ is below a threshold, $\hat{\lambda}$. This threshold does not depend on the proportion $1 / B$, it depends only on the maximum fraud level $\bar{\alpha}$.

Regarding a cooperative of myopic agents with same micro-economic ratio, we have proved that the cooperative is never viable when the microeconomic ratio is above the same threshold value $\hat{\lambda}$. When it is lower than this threshold, we have proved that the cooperative is viable if and only if the initial price belongs to a specific interval.

Regarding a cooperative composed of myopic and maximizing agents with identical economic ratio and with an accurate perception of the other members' behavior, we have proved that when the maximizing strategy is not 
viable, then the myopic agents are no more viable either.

Regarding a cooperative composed of myopic agents and identical (same $K(0), w, C, B)$ pro-viable agents, with same micro-economic ratio $\frac{C}{w p_{0}}$ for both pro-viable and myopic type of agents, we have proved that the local viability kernel of pro-viable agents contains the viability area of the cooperative with only myopic agents.

Besides, we have illustrated by simulation the behavior of such a cooperative of myopic and pro-viable agents with an accurate perception of the other members' behavior. The simulation results show how in some cases pro-viable agents increase the viability area of myopic agents.

\section{Acknowledgment}

We acknowledge the support of Réseau National des Systèmes Complexes - Call for ideas 2010. 


\section{References}

[1] G. Hardin, The tragedy of the commons, Science 162 (3859) (1968) $1243-1248$.

[2] E. Ostrom, Governing the commons, the evolution of institutions for collective action. (Cambridge University Press, 1990).

[3] P. Schuster, The commons' tragicomedy: Self-governance doesn't come easily, Complexity 10 (6) (2005) 10-12.

[4] M.J. Wooldridge, An introduction to multi-agent systems (Wiley, 2009).

[5] F. Billari, T. Fent, A. Prskawetz and J. Scheffran, Agent Based Computational Modelling in Demography, Economic and Environmental Sciences (Springer, 2006).

[6] J. Scheffran and B. Hannon, From complex conflicts to stable cooperation: Cases in environment and security, Complexity 13 (2) (2007) 78-91.

[7] O. Lpez-Corona, P. Padilla, O. Escolero, F. Armas, R. Garca-Arrazola and R. Esparza, Playing with models and optimization to overcome the tragedy of the commons in groundwater, Complexity 19 (1) (2013) 9-21.

[8] J.-P. Aubin, Viability theory (Birkhaser, 1991).

[9] J.-P. Aubin, A. Bayen and P. Saint-Pierre, Viability Theory. New Directions. (Springer, 2011).

[10] V. Martinet, O. Thébaud, and L. Doyen, Defining viable recovery paths toward sustainable fisheries, Ecol. Econ. 64 (2) (2007) 411-422.

[11] C. Bene, L. Doyen and D. Gabay, A viability analysis for a bio-economic model, Ecol. Econ. 36 (2001) 385-396.

[12] L. Chapel, G. Deffuant, S. Martin and C. Mullon, Defining yield policies in a viability approach, Ecol. Model. 212 (1-2) (2008) 10-15.

[13] M. DeLara, L. Doyen, T. Guilbaud and M.J. Rochet, Is a management framework based on spawning-stock biomass indicators sustainable? a viability approach, J. Mar. Sci. 64 (2007) 761-767. 
[14] P. Andrés Domenech, P. Saint-Pierre and G. Zaccour, Forest conservation and co2 emissions: A viable approach, Environ. Model. Assess. 16 (2011) 519-539.

[15] C. Bernard and S. Martin, Comparing the sustainability of different action policy possibilities: Application to the issue of both household survival and forest preservation in the corridor of Fianarantsoa, Math. Biosci. () (to appear).

[16] S. Martin, The cost of restoration as a way of defining resilience: a viability approach applied to a model of lake eutrophication, Ecol. Soc. 9 (2) (2004).

[17] P. Cury, C. Mullon, S. Garcia and L.J. Shannon, Viability theory for an ecosystem approach to fisheries, J. Mar. Sci., 62 (3) (2005) 577-584.

[18] L. Doyen, M. DeLara, J. Ferraris and D. Pelletier, Sustainability of exploited marine ecosystems through protected areas: a viability model and a coral reef case study, Ecol. Model. 208 (2-4) (2007) 353-366.

[19] W. Wei, I. Alvarez and S. Martin, Sustainability analysis: viability concepts to consider transient and asymptotical dynamics, Ecol. Model. 251 (2013) 103-113.

[20] J. P. Aubin, N. Bonneuil, F. Maurin and P. Saint-Pierre, Viability of pay-as-you-go systems, J. Evol. Econ. 11 (5) (2001) 555-571.

[21] C. Bernard and S. Martin, Building strategies to ensure language coexistence in presence of bilingualism, Appl. Math. Comput. 218 (17) (2012) $8825-8841$.

[22] J.-C. Péreau, L. Doyen, L.R. Little, and O. Thébaud, The triple bottom line: Meeting ecological, economic and social goals with individual transferable quotas, J. Environ. Econ. Manag. (2012), to appear.

[23] L. Doyen and J.-C. Péreau, Sustainable coalitions in the commons, Math. Soc. Sci. 63 (1) (2012) 57-64.

[24] F. Schweitzer, P. Mavrodiev and C. Tessone, How can social herding enhance cooperation?, Adv. Complex Syst. (2) (2013) 1-22. 
[25] D. Helbing and A. Johansson, Cooperation, norms, and revolutions: A unified game-theoretical approach, PLoS ONE 5 (10) (2010) art. no. e12530.

[26] P. Bettiol, P. Cardaliaguet and M. Quincampoix, Zero-sum state constraint differential game: Existence of a value for Bolza problem, . Int. J. Game Theory 34 (3) (2006) 495-527.

[27] J.-M. Lasry and P.-L. Lions, Mean field games Jpn. J. Math. 2 (1) (2007) 229-260.

[28] A. Bensoussan and J. Frehse, Control and nash games with mean field effect Chin. Ann. Math.. Ser. B 34 (2) (2013) 161-192.

[29] R. Buckdahn, P. Cardaliaguet and M. Quincampoix, Some recent aspects of differential game theory Dyn. Games Appl. 1 (1) (2011) 74-114.

[30] G. Aschhoff and E. Henningsen, The German Cooperative System (Publications of the DG Bank, Frankfurt am Main, 1986).

[31] J. Nilsson, The emergence of new organizational models for agricultural cooperatives, Swed. J. Agric. Res. 28 (1) (1998) 39-47.

[32] M. Fulton and K. Giannakas, Organizational Commitment in a Mixed Oligopoly: Agricultural Cooperatives and Investor-Owned Firms, Am. J. Agric. Econ. 83 (2001) 1258-1265.

[33] Y. Chiffoleau, F. Dreyfus and J.-M. Touzard, Ethics in French wine cooperatives : part of a social movement?, Creating food futures : trade, ethics and the environment (Gower Applied Business Research, 2008).

[34] M. L. Cook, F. R. Chaddad and C. Iliopoulos, Advances in Cooperative Theory since 1990: A Review of Agricultural Economics Literature, Restructuring Agricultural Cooperatives (Erasmus University Rotterdam, Rotterdam School of Management, 2004).

[35] P. Saint-Pierre, Approximation of the viability kernel, Appl. Math. Opt. 29 (1994) 187-209. 


\section{Appendix A. Proof of theorem 1}

We recall the statement of the theorem :

Let $f:] 0 ; 2] \rightarrow \mathbb{R}$ such that $f(A)=\frac{(1+A)^{2}}{4 A}$ if $A \leq 1$ and $f(A)=1$ otherwise.

$\operatorname{Viab}_{(14)}(L) \neq \emptyset$ if and only if $\frac{C}{p_{0} w} \leq f(A)$.

We recall that the equation which governs the evolution of $K$ according to $p$ and the variation of $p, \Delta p$, is :

$$
\begin{aligned}
\Delta K(p, \Delta p)= & -\frac{B D w}{p_{0} A} \Delta p^{2}+\frac{w}{p_{0} A}\left(p_{0}(1+A)-p(1+B D)\right) \Delta p+\frac{p w}{p_{0} A}\left(p_{0}(1+A)-p\right)-C \\
& \text { if } \Delta p \geq 0 \\
\Delta K(p, \Delta p)= & -\frac{B w}{p_{0} A} \Delta p^{2}+\frac{w}{p_{0} A}\left(p_{0}(1+A)-p(1+B)\right) \Delta p+\frac{p w}{p_{0} A}\left(p_{0}(1+A)-p\right)-C \\
& \text { if } \Delta p \leq 0 .
\end{aligned}
$$

where $A=\frac{\bar{\alpha}+1}{\bar{\alpha}^{2}}>0, D:=1 /(1-\delta) \geq 1$ since $\delta$ which measures the influence of reputation belongs to $[0 ; 1[$, and $B>1$ is the inverse of the production proportion $1 / B$ that experiences the new fraud level, whereas the remaining proportion, $1-1 / B$ experiences a fraud level equal to the previous fraud average.

We also recall that $c(p):=\Delta K(p, 0)$ and :

$$
c(p):=\frac{p w}{p_{0} A}\left(p_{0}(1+A)-p\right)-C
$$

Moreover, when $u$ ranges from 1 to $\bar{\alpha}, \Delta p$ ranges from $\Delta p_{\min }(p)$ to $\Delta p_{\max }(p)$ with, from (14),

$$
\Delta p_{\min }(p)=1 / B\left(p_{0} / \bar{\alpha}^{2}-p\right)
$$

and

$$
\Delta p_{\max }(p)=1 /(B D)\left(p_{0}-p\right)
$$

Proof. Let $\tilde{C}=\max _{p \in\left[\frac{p_{0}}{\alpha^{2}} ; p_{0}\right]} c(p)$ and $\tilde{p}=\operatorname{argmax}_{p \in\left[\frac{p_{0}}{\alpha^{2}} ; p_{0}\right]} c(p)$.

If the condition of the theorem is satisfied, that is $\frac{C}{p_{0} w} \leq f(A)$, then $\tilde{C}$ is positive.

Let define the evolution $(K(n), p(n)), n \in \mathbb{N}$ governed by (14) with $K(0)=$ $K_{\text {min }}$ and $p(0)=\tilde{p}$ and $\forall n, u(n)=\frac{1}{p_{0} A}\left(p_{0}(1+A)-p(0)\right)$ such that $p(n+1)=$ $p(n)=p(0)=\tilde{p}$ from $(19)$. 
Then, $\forall n, K(n+1)-K(n)=\Delta K(\tilde{p}, 0)=c(\tilde{p})=\tilde{C} \geq 0$.

Consequently, $\forall n, K(n) \geq K(0)=K_{\text {min }}$. The evolution $(K(n), p(n))$ remains in the constraint set $L,\left(K_{m i n}, \tilde{p}\right) \in \operatorname{Viab}_{(14)}(L)$ and $\operatorname{Viab}_{(14)}(L) \neq \emptyset$.

If the condition of the theorem is not satisfied, $\frac{C}{p_{0} w}>f(A)$, then $\tilde{C}<0$ and $\forall p \in\left[\frac{p_{0}}{\alpha^{2}} ; p_{0}\right], \Delta K(p, 0)=c(p) \leq \tilde{C}<0$. Moreover, since the parabola which represents $\Delta K(p,$.$) as a function of \Delta p$ is located below its tangents, from (A.1), $\Delta K(p, \Delta p)$ remains lower than $\tilde{C} / 2$ when

$$
|\Delta p| \leq \frac{|\tilde{C}|}{2\left|\frac{w}{p_{0} A}\left(p_{0}(1+A)-p(1+B D)\right)\right|} .
$$

Since function $p \rightarrow\left|\frac{w}{p_{0} A}\left(p_{0}(1+A)-p(1+B D)\right)\right|$ is bounded on $\left[\frac{p_{0}}{\alpha^{2}} ; p_{0}\right]$, there exists $\tilde{\Delta p}>0$ such that, for all $p \in\left[\frac{p_{0}}{\alpha^{2}} ; p_{0}\right],|\Delta p| \leq \tilde{\Delta p}$ implies $\Delta K(p, \Delta p)<\tilde{C} / 2$ :

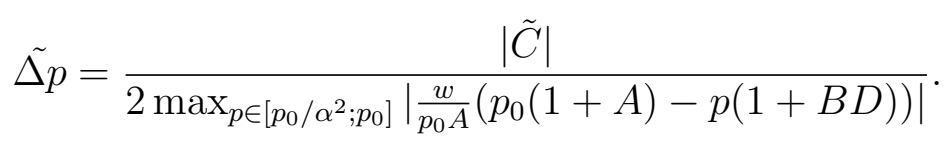

Let define $S$ by:

$$
S:=\left\{(\Delta, p) \in \mathbb{R}^{+} \times\left[\frac{p_{0}}{\alpha^{2}} ; p_{0}\right] \mid \Delta>0 \text { and } p+\Delta \leq p_{0} \text { and } p-\Delta \geq \frac{p_{0}}{\alpha^{2}}\right\},
$$

For all pairs $(\Delta, p) \in S$, we consider all pairs $\left(p_{1}, \Delta p_{1}\right) \in S_{1}(\Delta, p)$ where

$$
\begin{aligned}
S_{1}(\Delta, p):=\left\{\left(p_{1}, \Delta p_{1}\right) \in[\right. & {\left[\frac{p_{0}}{\alpha^{2}} ; p_{0}\right] \times\left[0 ; \Delta p_{\max }\left(p_{1}\right)\right] \mid } \\
& \left.p_{1} \leq p-\Delta \text { and } p_{1}+\Delta p_{1} \geq p+\Delta\right\}
\end{aligned}
$$

More precisely, from (A.4), $p_{1} \in\left[\max \left(\frac{p 0}{\alpha^{2}}, \frac{p+\Delta-p_{0} /(B D)}{1-1 /(B D)}\right), p-\Delta\right]$ and $\Delta p_{1} \in$ $\left[p+\Delta-p_{1} ; 1 /(B D)\left(p_{0}-p_{1}\right)\right]$ (Figure A.11).

We now aim at maximizing $\frac{\Delta K\left(p_{1}, \Delta p_{1}\right)}{\Delta p_{1}}$, the marginal capital growth, for $\left(p_{1}, \Delta p_{1}\right) \in S_{1}(\Delta, p)$.

$$
\frac{\Delta K\left(p_{1}, \Delta p_{1}\right)}{\Delta p_{1}}=-\frac{B D w}{p_{0} A} \Delta p_{1}+\frac{w}{p_{0} A}\left(p_{0}(1+A)-p_{1}(1+B D)\right)+\frac{\frac{p_{1} w}{p_{0} A}\left(p_{0}(1+A)-p_{1}\right)-C}{\Delta p_{1}}
$$




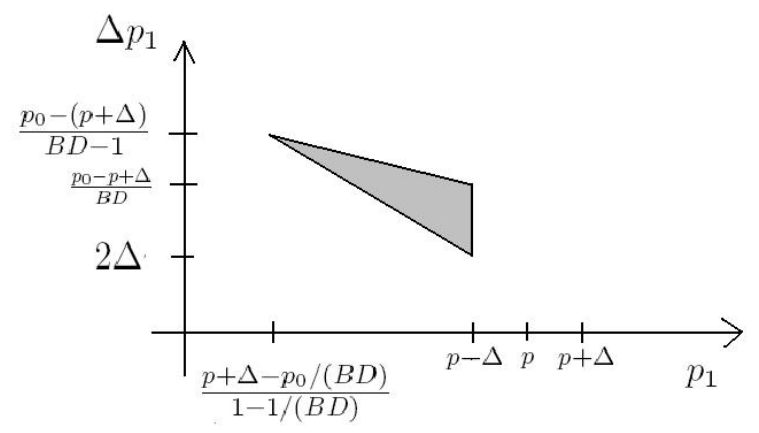

Figure A.11: The area $S_{1}(\Delta, p)$ is colored grey.

We differentiate $\frac{\Delta K\left(p_{1}, \Delta p_{1}\right)}{\Delta p_{1}}$ according to $p_{1}$ and $\Delta p_{1}$ :

$$
\frac{\partial}{\partial \Delta p_{1}} \frac{\Delta K\left(p_{1}, \Delta p_{1}\right)}{\Delta p_{1}}=-\frac{B D w}{p_{0} A}+\frac{C-\frac{p_{1} w}{p_{0} A}\left(p_{0}(1+A)-p_{1}\right)}{\Delta p_{1}^{2}} .
$$

Then,

$$
\frac{\partial}{\partial \Delta p_{1}} \frac{\Delta K\left(p_{1}, \Delta p_{1}\right)}{\Delta p_{1}}=0 \Leftrightarrow \Delta p_{1}^{2}=1 /(B D)\left(p_{1}^{2}-p_{0}(1+A) p_{1}+\frac{p_{0} A C}{w}\right) .
$$

Moreover,

$$
\frac{\partial}{\partial p_{1}} \frac{\Delta K\left(p_{1}, \Delta p_{1}\right)}{\Delta p_{1}}=-\frac{w}{p_{0} A}(1+B D)+\frac{w}{A \Delta p_{1}}(1+A)-\frac{2 w}{p_{0} A \Delta p_{1}} p_{1} .
$$

Then,

$$
\frac{\partial}{\partial p_{1}} \frac{\Delta K\left(p_{1}, \Delta p_{1}\right)}{\Delta p_{1}}=0 \Leftrightarrow \Delta p_{1}=1 /(1+B D)\left(p_{0}(1+A)-2 p_{1}\right) .
$$

Thus,

$$
\frac{\partial}{\partial \Delta p_{1}} \frac{\Delta K\left(p_{1}, \Delta p_{1}\right)}{\Delta p_{1}}=\frac{\partial}{\partial p_{1}} \frac{\Delta K\left(p_{1}, \Delta p_{1}\right)}{\Delta p_{1}}=0
$$

amounts to

$$
\left(1 /(1+B D)\left(p_{0}(1+A)-2 p_{1}\right)\right)^{2}=1 /(B D)\left(p_{1}^{2}-p_{0}(1+A) p_{1}+\frac{p_{0} A C}{w}\right)
$$

and

$$
-(B D-1)^{2} p_{1}^{2}+p_{0}(1+A)(B D-1)^{2} p_{1}+p_{0}\left(p_{0}(1+A)^{2} B D-(1+B D)^{2} A C / w\right)=0 .
$$


Let $g\left(p_{1}\right)=-(B D-1)^{2} p_{1}^{2}+p_{0}(1+A)(B D-1)^{2} p_{1}+p_{0}\left(p_{0}(1+A)^{2} B D-\right.$ $\left.(1+B D)^{2} A C / w\right)$. The top of the parabola $g$ is reached for $p_{0} \frac{1+A}{2}$.

Since $\frac{C}{p_{0} w}>f(A)$,

- case 1: $A \leq 1$, then the discriminant of the quadratic trinomial, $g$, equals

$p_{0}^{2}(1+A)^{2}(B D-1)^{4}+4(B D-1)^{2} p_{0}\left(p_{0}(1+A)^{2} B D-(1+B D)^{2} A C / w\right)$

and is strictly negative since $\frac{C}{p_{0} w}>\frac{(1+A)^{2}}{4 A}$, which implies

$$
p_{0}(1+A)^{2} B D-(1+B D)^{2} A C / w<-p_{0} \frac{(1+A)^{2}}{4}(B D-1)^{2} .
$$

Consequently, $\frac{\Delta K\left(p_{1}, \Delta p_{1}\right)}{\Delta p_{1}}$ does not reach any local maximum in the interior of $S_{1}(\Delta, p)$,

- case $2: A \geq 1$, then $g$ reaches its maximum for $p_{1}=p_{0} \frac{1+A}{2}>p_{0}$. The discriminant of $g$ can be positive, but then the $p_{1}$-value for which $g$ is null corresponds to a local minimum of $\frac{\Delta K\left(p_{1}, \Delta p_{1}\right)}{\Delta p_{1}}$ and again, $\frac{\Delta K\left(p_{1}, \Delta p_{1}\right)}{\Delta p_{1}}$ does not reach any local maximum in the interior of $S_{1}(\Delta, p)$.

Consequently, the maximum of $\frac{\Delta K\left(p_{1}, \Delta p_{1}\right)}{\Delta p_{1}}$ for $\left(p_{1}, \Delta p_{1}\right) \in S_{1}(\Delta, p)$ belongs to the boundary of the domain $S_{1}(\Delta, p)$ (Figure A.11).

On the upper boundary, $\Delta p_{1}=1 /(B D)\left(p_{0}-p_{1}\right)$, then,

$$
\frac{\Delta K\left(p_{1}, 1 /(B D)\left(p_{0}-p_{1}\right)\right)}{1 /(B D)\left(p_{0}-p_{1}\right)}=w \frac{p_{1}(B D-1)+p_{0}-C B D / w}{p_{0}-p_{1}}
$$

and

$$
\begin{aligned}
\frac{d}{d p_{1}} \frac{\Delta K\left(p_{1}, 1 /(B D)\left(p_{0}-p_{1}\right)\right)}{1 /(B D)\left(p_{0}-p_{1}\right)} & =w B D \frac{p_{0}-C / w}{\left(p_{0}-p_{1}\right)^{2}} \\
& =\frac{B D}{\left(p_{0}-p_{1}\right)^{2}} c\left(p_{0}\right)<0 .
\end{aligned}
$$

So $\frac{\Delta K\left(p_{1}, 1 /(B D)\left(p_{0}-p_{1}\right)\right)}{1 /(B D)\left(p_{0}-p_{1}\right)}$ decreases with $p_{1}$ on the upper boundary of $S_{1}(\Delta, p)$. On the lower boundary, $\Delta p_{1}=p+\Delta-p_{1}$,

$$
\frac{\Delta K\left(p_{1}, p+\Delta-p_{1}\right)}{p+\Delta-p_{1}}=\frac{w}{p_{0} A} \frac{\left(p_{1}(B D-1)+p_{0}(1+A)\right)(p+\Delta)-B D(p+\Delta)^{2}-C}{p+\Delta-p_{1}}
$$

and

$$
\begin{aligned}
\frac{d}{d p_{1}} \frac{\Delta K\left(p_{1}, p+\Delta-p_{1}\right)}{p+\Delta-p_{1}} & =\frac{w}{A p_{0}} \frac{-(p+\Delta)^{2}+p_{0}(1+a)(p+\Delta)-C}{\left(p+\Delta-p_{1}\right)^{2}} \\
& =\frac{1}{\left(p+\Delta-p_{1}\right)^{2}} c(p+\Delta)<0 .
\end{aligned}
$$


So $\frac{\Delta K\left(p_{1}, p+\Delta-p_{1}\right)}{p+\Delta-p_{1}}$ decreases with $p_{1}$ on the lower boundary of $S_{1}(\Delta, p)$.

Moreover, for all $\hat{p} \in\left[p+\Delta ; p+\Delta+1 /(B D)\left(p_{0}-p+\Delta\right)-2 \Delta\right]$,

$$
\begin{aligned}
\frac{d}{d p_{1}} \frac{\Delta K\left(p_{1}, \hat{p}-p_{1}\right)}{\hat{p}-p_{1}} & =\frac{w}{A p_{0}} \frac{-\hat{p}^{2}+p_{0}(1+a) \hat{p}-C}{\left(\hat{p}-p_{1}\right)^{2}} \\
& =\frac{1}{\left(\hat{p}-p_{1}\right)^{2}} c(\hat{p})<0 .
\end{aligned}
$$

Thus,

$$
\begin{aligned}
\max _{\left(p_{1}, \Delta p_{1}\right) \in S_{1}(\Delta, p)} \frac{\Delta K\left(p_{1}, \Delta p_{1}\right)}{\Delta p_{1}} & \leq \frac{\Delta K\left(\frac{p+\Delta-p_{0} /(B D)}{1-1 /(B D)}, \frac{p_{0}-(p+\Delta)}{B D-1}\right)}{\frac{p_{0}-(p+\Delta)}{B D-1}} \\
& \leq w \frac{\frac{p+\Delta-p_{0} /(B D)}{1-1 /(B D)}(B D-1)+p_{0}-C B D / w}{p_{0}-\frac{p+\Delta-p_{0} /(B D)}{1-1 /(B D)}} \\
& \leq w(B D-1) \frac{p+\Delta-\frac{C}{w}}{p_{0}-(p+\Delta)}
\end{aligned}
$$

Moreover,

$$
\frac{\partial\left(\frac{p+\Delta-\frac{C}{w}}{p_{0}-(p+\Delta)}\right)}{\partial \Delta}(\Delta, p)=\frac{p_{0}-\frac{C}{w}}{\left(p_{0}-(p+\Delta)\right)^{2}}<0
$$

when $\frac{C}{p_{0} w}>f(A)$.

So, let define $S(p):=\{\Delta$ such that $(p, \Delta) \in S\}$,

$$
\begin{aligned}
\max _{\substack{\Delta \in S(p) \\
\left(p_{1}, \Delta p_{1}\right) \in S_{1}(\Delta, p)}} \frac{\Delta K\left(p_{1}, \Delta p_{1}\right)}{\Delta p_{1}} & \leq w(B D-1) \lim _{\Delta \rightarrow 0^{+}}\left(\frac{p+\Delta-\frac{C}{w}}{p_{0}-(p+\Delta)}\right) \\
& \leq w(B D-1) \frac{p-\frac{C}{w}}{p_{0}-p} \\
& \leq w(B-1) \frac{p-\frac{C}{w}}{p_{0}-p}:=f_{1}(p) .
\end{aligned}
$$

$f_{1}(p)$ is defined for $p \in\left[p_{0} / \alpha^{2} ; p_{0}\left[\right.\right.$ and admits an upper bound, $\max _{p \in\left[p_{0} / \alpha^{2} ; p_{0}[\right.} f_{1}(p) \leq$ $F_{1}<+\infty$.

Given $(\Delta, p) \in S$, let us now consider all pairs $\left(p_{2}, \Delta p_{2}\right) \in S_{2}$, where

$$
\begin{aligned}
S_{2}(\Delta, p):=\left\{\left(p_{2}, \Delta p_{2}\right) \in\right. & {\left[\frac{p_{0}}{\alpha^{2}} ; p_{0}\right] \times\left[\Delta p_{\min }\left(p_{2}\right) ; 0\right] } \\
& \left.p_{2} \geq p+\Delta \text { and } p_{2}+\Delta p_{2} \leq p-\Delta\right\} .
\end{aligned}
$$

More precisely, $p_{2} \in\left[p+\Delta ; \min \left(p_{0}, \frac{p-\Delta-p_{0} /\left(B \alpha^{2}\right)}{1-1 / B}\right)\right]$ and $\Delta p_{2} \in\left[1 / B\left(p_{0} / \alpha^{2}-\right.\right.$ $\left.\left.p_{2}\right) ; p-\Delta-p_{2}\right]$. 
We now aim at minimizing $\frac{\Delta K\left(p_{2}, \Delta p_{2}\right)}{\Delta p_{2}}$, the marginal capital growth, for $\left(p_{2}, \Delta p_{2}\right) \in S_{2}(\Delta, p)$.

Following the same reasoning as above, we show that

$$
\min _{\left(p_{2}, \Delta p_{2}\right) \in S_{2}(\Delta, p)} \frac{\Delta K\left(p_{2}, \Delta p_{2}\right)}{\Delta p_{2}} \geq \frac{\Delta K\left(\frac{p-\Delta-p_{0} /\left(B \alpha^{2}\right)}{1-1 / B}, \frac{p_{0} / \alpha^{2}-(p-\Delta)}{B-1}\right)}{\frac{p_{0} / \alpha^{2}-(p-\Delta)}{B-1}}
$$

for all $(\Delta, p) \in S$.

On the upper boundary of $S_{2}, \Delta p_{2}=1 / B\left(\frac{p_{0}}{\alpha^{2}}-p_{2}\right)$, then,

$$
\frac{\Delta K\left(p_{2}, 1 / B\left(\frac{p_{0}}{\alpha^{2}}-p_{2}\right)\right)}{1 / B\left(\frac{p_{0}}{\alpha^{2}}-p_{2}\right)}=\alpha w \frac{p_{2}(B-1)+\frac{p_{0}}{\alpha^{2}}-C B /(\alpha w)}{\frac{p_{0}}{\alpha^{2}}-p_{2}}
$$

Then,

$$
\min _{\left(p_{2}, \Delta p_{2}\right) \in S_{2}(\Delta, p)} \frac{\Delta K\left(p_{2}, \Delta p_{2}\right)}{\Delta p_{2}} \geq w(B-1) \frac{\alpha(p-\Delta)-\frac{C}{w}}{p_{0} / \alpha^{2}-(p-\Delta)}
$$

Since $\frac{\alpha(p-\Delta)-\frac{C}{w}}{p_{0} / \alpha^{2}-(p-\Delta)}$ increases with $\Delta$,

$$
\begin{aligned}
\min _{\substack{\left.\Delta \in S(p) \\
\Delta p_{2}\right) \in S_{2}(\Delta, p)}} \frac{\Delta K\left(p_{2}, \Delta p_{2}\right)}{\Delta p_{2}} & \geq w(B-1) \lim _{\Delta \rightarrow 0^{+}}\left(\frac{\alpha(p-\Delta)-\frac{C}{w}}{p_{0} / \alpha^{2}-(p-\Delta)}\right) \\
& \geq w(B-1) \frac{\alpha p-\frac{C}{w}}{p_{0} / \alpha^{2}-p}:=f_{2}(p)
\end{aligned}
$$

$f_{2}(p)$ is defined for $\left.\left.p \in\right] p_{0} / \alpha^{2} ; p_{0}\right]$ and admits a lower bound, $\max _{\left.p \in] p_{0} / \alpha^{2} ; p_{0}\right]} f_{2}(p) \geq$ $F_{2}>-\infty$.

Moreover,

$$
\begin{aligned}
f_{1}(p)-f_{2}(p) & \leq w(B-1)\left[\frac{p-C / w}{p_{0}-p}-\frac{\alpha p-C / w}{p_{0} / \alpha^{2}-p}\right] \\
& \leq w(B-1) \frac{(1-\alpha) p^{2}+p p_{0}\left(\alpha-1 / \alpha^{2}\right)+C p_{0} / w\left(1 / \alpha^{2}-1\right)}{\left(p_{0}-p\right)\left(p-p_{0} / \alpha^{2}\right)} \\
& \leq w(B-1)(\alpha-1) \frac{-p^{2}+p p_{0}(1+A)-C p_{0} A / w}{\left(p_{0}-p\right)\left(p-p_{0} / \alpha^{2}\right)} \\
& \leq w(B-1)(\alpha-1) \frac{p_{0} A / w c(p)}{\left(p_{0}-p\right)\left(p-p_{0} / \alpha^{2}\right)} \\
& \leq p_{0} A(B-1)(\alpha-1) \frac{\tilde{C}}{\left(p_{0}-p\right)\left(p-p_{0} / \alpha^{2}\right)} \\
& \leq 4 \frac{B-1}{p_{0} A(\alpha-1)} \tilde{C} \\
& <0 .
\end{aligned}
$$


Let $(K(n), p(n)), n \in \mathbb{N}$ an evolution governed by (14), we aim at showing that $K(n)$ is lower than a sequence which tends toward $-\infty$ when $n$ goes to infinity.

If $p(i+1)-p(i)=0, K(i+1)-K(i)=\Delta K(p(i), 0)=c(p(i))<0$ since $\frac{C}{p_{0} w}<f(A)$, so we consider the more favourable case where $\forall i p(i+1)-p(i) \neq$ 0 .

$$
\begin{aligned}
& K(n)=K(0)+\sum_{i=0}^{n-1} K(i+1)-K(i) \\
&=K(0)+\sum_{i=0}^{n-1} \int_{p(i)}^{p(i+1)} \frac{K(i+1)-K(i)}{p(i+1)-p(i)} d p \\
& \text { If } p(i+1)-p(i)>0, \frac{K(i+1)-K(i)}{p(i+1)-p(i)} \leq \max _{\substack{\Delta \in S(p) \\
\left(p_{1}, \Delta p_{1}\right) \in S_{1}(\Delta, p)}} \frac{\Delta K\left(p_{1}, \Delta p_{1}\right)}{\Delta p_{1}} \leq f_{1}(p) \text {, for }
\end{aligned}
$$

all $p \in[p(i) ; p(i+1)]$.

$$
\text { If } p(i+1)-p(i)<0, \frac{K(i+1)-K(i)}{p(i+1)-p(i)} \geq \min _{\substack{\Delta \in S(p) \\\left(p_{2}, \Delta p_{2}\right) \in S_{2}(\Delta, p)}} \frac{\Delta K\left(p_{2}, \Delta p_{2}\right)}{\Delta p_{2}} \geq f_{2}(p) \text {, for all }
$$

$p \in[p(i) ; p(i+1)]$.

\section{Consequently,}

$$
\begin{aligned}
K(n) & =K(0)+\sum_{i=0}^{n-1} \int_{p(i)}^{p(i+1)} \frac{K(i+1)-K(i)}{p(i+1)-p(i)} d p \\
& =K(0)+\sum_{p(i+1)-p(i)>0}^{n-1} \int_{p(i)}^{p(i+1)} \frac{K(i+1)-K(i)}{p(i+1)-p(i)} d p+\sum_{p(i+1)-p(i)<0}^{n-1} \int_{p(i)}^{p(i+1)} \frac{K(i+1)-K(i)}{p(i+1)-p(i)} d p \\
& =K(0)+\sum_{p(i+1)-p(i)>0}^{n-1} \int_{p(i)}^{p(i+1)} \frac{K(i+1)-K(i)}{p(i+1)-p(i)} d p+\sum_{p(i+1)-p(i)<0}^{n-1} \int_{p(i+1)}^{p(i)}-\frac{K(i+1)-K(i)}{p(i+1)-p(i)} d p \\
& \leq K(0)+\sum_{p(i+1)-p(i)>0}^{n-1} \int_{p(i)}^{p(i+1)} f_{1}(p) d p+\sum_{p(i+1)-p(i)<0}^{n-1} \int_{p(i+1)}^{p(i)}-f_{2}(p) d p
\end{aligned}
$$

Assume for instance that $p(n) \geq p(0)$,

$$
\begin{aligned}
K(n) & \leq K(0)+\sum_{p(i+1)-p(i)<0}^{n-1} \int_{p(i+1)}^{p(i)}\left(f_{1}(p)-f_{2}(p)\right) d p+\int_{p(0)}^{p(n)} f_{1}(p) d p \\
& \leq \sum_{\substack{i=0 \\
p(i+1)-p(i)<0}}^{p(i+1)} \frac{4(B-1)}{p_{0} A(\alpha-1)} \tilde{C} d p+(p(n)-p(0)) \max _{p \in\left[p_{0} / \alpha^{2} ; p_{0}[\right.} f_{1}(p) \\
& \leq \frac{4(B-1)}{p_{0} A(\alpha-1)} \tilde{C} \frac{1}{2}\left[\sum_{i=0}^{n-1}|p(i+1)-p(i)|-(p(n)-p(0))\right]+\left(p_{0}-p_{0} / \alpha^{2}\right) F_{1}
\end{aligned}
$$




$$
\begin{aligned}
& \text { If } p(n) \leq p(0), \\
& \begin{aligned}
K(n) & \leq K(0)+\sum_{\substack{p(i+1)-p(i)>0 \\
n-1}}^{p} \int_{p(i)}^{p(i+1)}\left(f_{1}(p)-f_{2}(p)\right) d p+\int_{p(0)}^{p(n)}-f_{2}(p) d p \\
& \leq \frac{4(B-1)}{p_{0} A(\alpha-1)} \tilde{C} \frac{1}{2}\left[\sum_{i=0}^{n-1}|p(i+1)-p(i)|-(p(n)-p(0))\right]+\left(p_{0}-p_{0} / \alpha^{2}\right) F_{2} .
\end{aligned}
\end{aligned}
$$

Consequently, there exits $M \in \mathbb{R}$ such that :

$$
K(n) \leq K(0)+2 \sum_{j=1}^{n-1}\left|\Delta p_{j}\right| \frac{B-1}{p_{0} A(\alpha-1)} \tilde{C}+M
$$

If $\sum_{j=1}^{n-1}\left|\Delta p_{j}\right|$ tends toward $+\infty$ when $n$ goes to infinity, then $K(n)$ tends toward $-\infty$ since $\frac{B-1}{p_{0} A(\alpha-1)} \tilde{C}<0$ when $\frac{C}{p_{0} w}>f(A)$.

Otherwise, there exists $N \in \mathbb{N}$ such that, for all $n \geq N,|\Delta p(n)| \leq \tilde{\Delta p}$, and then from (A.6), $K(n+1)-K(n)=\Delta K(p(n), \Delta p(n))<\tilde{C} / 2$ and $K(n)$ tends toward $-\infty$.

In both cases $(K(n), p(n))$ does not remain in the constraint set $L$ and the viability kernel of $L$ for dynamics (14) when $\frac{C}{p_{0} w}>f(A)$ is then empty. 